\title{
ASSESSMENT OF PHYSICOCHEMICAL CHARACTERISTICS, OXIDATIVE, AND THERMAL PROPERTIES OF BUTTERS
}

\author{
Hasene Keskin Çavdar* \\ Department of Food Engineering, Faculty of Engineering, University of Gaziantep, Gaziantep, Turkey
}

Received /Geliş: 07.09.2021; Accepted / Kabul: 12.02.2022; Published online / Online bask1: 22.02.2022

Keskin Cavdar, H. (2022). Assessment of physicochemical characteristics, oxidative, and thermal properties of butters. GIDA 47 (2) 169-182 doi: 10.15237/gida.GD21121

Keskin Çavdar, H. (2022). Tereyağlarının fizikokimyasal, oksidatif ve termal özelliklerinin değerlendirilmesi. GIDA 47 (2) 169-182 doi: 10.15237/gida.GD21121

\begin{abstract}
This study aimed to evaluate the marketed butters in terms of physicochemical properties, oxidation levels, thermal behaviors, fatty acid composition, and quality matching of them with Turkish national standards. The mean value of investigated butters ( $\mathrm{n}=28$ ) was: moisture $20.67 \%$, fat $74.53 \%$, non-fat solids content $4.39 \%$, salt $0.19 \%$, lactic acid $0.65 \%$, oleic acid $1.55 \%(\mathrm{~m} / \mathrm{m})$, acid value $4.04 \mathrm{mg} \mathrm{KOH} / \mathrm{g}$ fat, peroxide value $3.25 \mathrm{meq} \mathrm{O}_{2} / \mathrm{kg}$ fat and thiobarbituric acid value $0.09 \mathrm{mg} \mathrm{MA} / \mathrm{kg}$ fat. The samples' average saturated, monounsaturated and polyunsaturated fatty acid contents were $68.30 \%, 27.90 \%$ and $2.98 \%$, respectively. The butters melted completely at a temperature varied from 28.58 to $36.80^{\circ} \mathrm{C}$. The solid fat content (SFC) of the butters was also investigated and the melting temperatures of the butters were aligned with SFC values. The results showed that examined butters did not completely agree with Turkish Food Regulations.

Keyword: Butter, physicochemical characteristics, oxidation, fatty acid composition

\section{TEREYAĞLARININ FİZIKOKİMYASAL, OKSİDATİF VE TERMAL ÖZELLİKLERINNIN DEĞERLENDİRİLMESİ}

\section{ÖZ}

Bu çalışma, piyasada satılan tereyağlarının fizikokimyasal özelliklerini, oksidasyon seviyelerini, termal davranışlarını, yağ asidi bileşimlerini ve Türk Gıda Kodeksi ile kalite uyumunu değerlendirmeyi amaçladi. İncelenen tereyağlarının ( $\mathrm{n}=28$ ) ortalama değerleri; nem $\% 20.67$, yağ $\% 74.53$, yağsız katı madde içeriği $\% 4.39$, tuz $\% 0.19$, laktik asit $\% 0.65$, oleik asit $\% 1.55(\mathrm{~m} / \mathrm{m})$, asit değeri $4.04 \mathrm{mg} \mathrm{KOH} / \mathrm{g}$ yağ , peroksit değeri 3.25 meq $\mathrm{O}_{2} / \mathrm{kg}$ yağ ve tiyobarbitürik asit değeri $0.09 \mathrm{mg} \mathrm{MA} / \mathrm{kg}$ yağ'dır. Örneklerin ortalama doymuş, tekli doymamış ve çoklu doymamış yağ asidi içerikleri sırasıyla $\% 68.30$, $\% 27.90$ ve $\% 2.98$ dir. Tereyağları $28.58-36.80{ }^{\circ} \mathrm{C}$ arasında değişen sıcaklıklarda tamamen eridi. Tereyağlarının katı yağ miktarları (KYM) incelendi ve erime sıcaklıkları ile KYM değerleri uyumlu bulundu. Sonuçlar, incelenen tereyağlarının Türk Gıda Mevzuatına tam olarak uymadığını gösterdi. Anahtar kelimeler: Tereyağı, fizikokimyasal karakteristikler, oksidasyon, yağ asidi kompozisyonu

${ }^{*}$ Corresponding author / Yazışmalardan sorumlu yazar:

$\varangle$ : hasenekeskin@gantep.edu.tr $\quad(0:$ (+90) 3423172319

冝: (+90) 3423172362

Hasene Keskin Çavdar ORCID ID: 0000-0001-5230-5904 


\section{INTRODUCTION}

There is an increasing demand for butter, especially for the positive health aspects. Butter contains many beneficial components for human health, such as lipid-soluble vitamins, antioxidants, and unsaturated fatty acids, and it is one of the most popular dairy products due to its aromatic characteristics and nutritive value (Akgül et al., 2021).

In 2020, the average worldwide butter production reached 12 million tons, increasing 1.8\% compared to the previous year. In the world butter market, $39.3 \%$ of the total production has been provided by India, while the EU provides $20.1 \%$. In Turkey, butter production has reached its maximum, 78.6 thousand tons, increasing $6.7 \%$ in 2020 (Anonymous, 2021a). High demand for butter, limited milk supply, and the high price of energy and labor causes high costs for butter (3200 USD/ton). Due to its relatively high price compared to other fats and oils, butter is often the object of fraud involving high water content or replacement with cheaper animal fats or plant oils (Tomaszewska-Gras, 2016). Adulteration has been performed mainly by adding plant oils, nonedible oils, used oils, or industrial creams to industrial or traditionally produced butters, impacting their quality and nutritional value (Koca et al., 2010).

One of the major problems regarding butter is deterioration (lipolysis and oxidation) caused by several factors such as long-term storage of butter. Deterioration causes flavor impairment, lowers nutritional quality, and creates severe problems for storage stability (Samet-Bali, Ayadi, \& Attia, 2009). Butter's quality and nutritional properties are also reduced during unsuitable storage, transportation conditions, and nonstandard production and packaging. Thus, designing appropriate storage regimes and suitable manufacturing is an essential issue for butter suppliers.

Most of the butters are usually sold in unsuitable containers, reused plastic, and do not have labels. Although the corresponding institutions regularly inspect the products, the butters without sufficient quality are sold in local or national supermarkets and bazaars. This research aimed to evaluate physicochemical, oxidative, and thermal properties of different butters and quality matching of them with Turkish national standards (Anonymous, 2021b).

\section{MATERIAL AND METHODS \\ Materials}

Different brands of butter $(\mathrm{n}=28)$ were supplied from National markets (B1-B18), local markets (B19-B23) and bazaars (B24-B28) on 23-24 June 2020 in Gaziantep, Turkey. All samples were stored at $4^{\circ} \mathrm{C}$ during the analysis period. All the chemicals and solvents were of analytical or chromatography grade and purchased from Sigma-Aldrich Co. (St. Louis, MO, USA).

\section{Butter oil extraction}

Butters were placed in a centrifuge tube and held at $50^{\circ} \mathrm{C}$ in a forced convection oven (JSOF-100, JS Research Inc., Korea) for $2 \mathrm{~h}$ to separate the butter oil from the butter. At the end of $2 \mathrm{~h}$, the samples were centrifuged at $6000 \mathrm{rpm}$ for $3 \mathrm{~min}$. Then, the samples were held again at $50^{\circ} \mathrm{C}$ for 15 min. Finally, clear butter oils were decanted and subjected to analysis.

\section{Physicochemical analyses \\ Moisture content}

The moisture content of butters was determined using the method suggested by AOAC (1998). The butters were dried to constant weight at 100 ${ }^{\circ} \mathrm{C}$ in a forced convection oven (JSOF-100, JS Research Inc., Korea) for $3 \mathrm{~h}$.

\section{Fat content}

Fat content of the butters was determined using the method suggested by AOAC (1998). Dried butter obtained after moisture content analysis was extracted using $n$-hexane in Soxhlet apparatus for $3 \mathrm{~h} . n$-hexane was then removed at $50{ }^{\circ} \mathrm{C}$ using a rotary vacuum evaporator (Heidolph Instrument GmbH \& Co .KG. Schwabach, Germany). The obtained oils were weighed to calculate the fat content and stored at $4{ }^{\circ} \mathrm{C}$ for further analysis. The fat content was calculated using equation 1 :

Fat content $\%(g / g)=\frac{\text { weight of extracted fat }(g)}{\text { weight of butter }(g)} \times 100$

(Equation 1) 


\section{Solids non-fat (SNF)}

Solids non-fat of butter was calculated according to the method recommended by Anonymous (2001). $10 \mathrm{~g}$ of butter was dissolved in $20 \mathrm{~mL}$ of petroleum ether. The mixture was transferred to the Gooch crucible and allowed to filter into the suction flask. This process was repeated five times. The sediment in the crucible was washed with $20 \mathrm{~mL}$ of petroleum ether. Finally, the Gooch crucible was dried at $102^{\circ} \mathrm{C}$ for $2 \mathrm{~h}$ until constant weight. Solids non-fat was calculated using the Equation 2:

Solid Non - Fat $(\%)=$

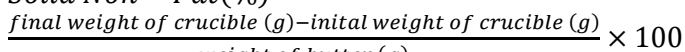

(Equation 2)

\section{Salt content}

The salt content of butter was calculated according to the method recommended by AOAC (1998). According to this method, $5 \mathrm{~g}$ of butter and $100 \mathrm{~mL}$ boiling distilled water were mixed and swirled occasionally for 5 minutes and cooled to $50-55^{\circ} \mathrm{C}$. Then, $2 \mathrm{~mL}$ of $\mathrm{K}_{2} \mathrm{CrO}_{4}$ was added to the mixture and the solution was titrated with $0.1 \mathrm{~N} \mathrm{AgNO}_{3}$. The salt content was calculated using Equation (3):

Salt $(\%, g / g)=\frac{\text { used } 0.1 \mathrm{NAgNO}_{3}(\mathrm{~mL}) \times 0.5844}{\text { weight of butter }(\mathrm{g})}$

(Equation 3)

\section{Acid value (AV) and titratable acidity}

Acid value and titratable acidity (lactic acid (LA), oleic acid $(\mathrm{OA}) \%$ ) of butter oil was calculated according to the method recommended by AOAC (1998). $2 \mathrm{~g}$ of butter oil was mixed with 15 $\mathrm{mL}$ ethanol and $10 \mathrm{~mL}$ diethyl ether. The solution was titrated with $0.1 \mathrm{~N} \mathrm{KOH}$. The acid value and titratable acidity in terms of lactic acid (LA) and oleic acid $(\mathrm{OA})$ were calculated using Equation 4, 5 and 6 , respectively.

$A V(m g \mathrm{KOH} / \mathrm{g}$ fat $)=$

used $\mathrm{KOH}$ solution $(\mathrm{mL}) \times$ normality $\mathrm{KOH} \times 56.1$ weight of butter oil $(g)$

(Equation 4)
$L A \%(g / g)=$

$\frac{\text { used } \mathrm{KOH} \text { solution }(\mathrm{mL}) \times \text { normality } \mathrm{KOH} \times 0.09}{\text { weight of butter oil }(\mathrm{g})} \times 100$

(Equation 5)

$O A \%(g / g)=$

$\frac{\text { used } \mathrm{KOH} \text { solution }(\mathrm{mL}) \times \text { normality } \mathrm{KOH} \times 0.28}{\text { weight of butter oil }(g)} \times 100$

(Equation 6)

\section{Peroxide value (PV)}

The peroxide value was determined following the AOCS (1997) with some modifications. $1 \mathrm{~mL}$ of saturated KI was added to $5 \mathrm{~g}$ of butter oils. The mixture was dissolved in $25 \mathrm{~mL}$ of acetic acid: chloroform $(3: 2, \mathrm{v} / \mathrm{v})$. The solution was slightly agitated for $5 \mathrm{~min}$ and kept for $5 \mathrm{~min}$ in the dark. Then, $75 \mathrm{~mL}$ of distilled water and $1 \mathrm{~mL}$ starch solution $(1 \%, \mathrm{~m} / \mathrm{m})$ were added to the mixture, respectively. Finally, liberated iodine was titrated with $0.01 \mathrm{~N} \mathrm{Na}_{2} \mathrm{~S}_{2} \mathrm{O}_{3}$. The peroxide value was calculated according to equation 6 and expressed as milliequivalents $\mathrm{O}_{2} / \mathrm{kg}$.

$\mathrm{PV}\left(\right.$ meq $\mathrm{O}_{2} / \mathrm{kg}$ fat $)=$

volume of sodium thiosulfate used mL $\times$ Normality of $\mathrm{Na}_{2} \mathrm{~S}_{2} \mathrm{O}_{3} \times 1000$ weight of the butter oil (g)

(Equation 7)

\section{Thiobarbituric acid (TBA)}

TBA value of butters was calculated following the method suggested by Asha et al. (2015). $0.1 \mathrm{~g}$ of butter fat mixed with trichloroacetic acid $(1 \mathrm{~mL})$ and TBA reagent $(2 \mathrm{~mL})$. After holding the mixture at boiling water bath for $15 \mathrm{~min}, 1 \mathrm{~mL}$ of glacial acetic acid and $2 \mathrm{~mL}$ of chloroform were added. The mixture was vortexed and centrifuged at $6000 \mathrm{rpm}$ for $5 \mathrm{~min}$. The absorbance of supernatant layer was read at $532 \mathrm{~nm}$ using a spectrophotometer. Blank was also prepared without sample. Concentrations were calculated using 1,1,3,3-tetra ethoxy propane within the range from 0 to $0.24 \mu \mathrm{g} / \mathrm{mL}$. Results were given as $\mathrm{mg}$ of malonaldehyde (MA)/ $\mathrm{kg}$ butter).

\section{Thermal analysis by DSC}

The melting profile and solid fat content (SFC) of butter oils were determined by DSC (Perkin Elmer DSC-6, Norwalk, CN, USA). Butter oil was heated at $80^{\circ} \mathrm{C}$ and then weighed $(5 \mathrm{mg})$ into an aluminum pan which was then sealed. In the DSC 
method, the sample was held at $80^{\circ} \mathrm{C}$ for $10 \mathrm{~min}$. Then, the temperature was decreased to $-60^{\circ} \mathrm{C}$ at a rate of $5^{\circ} \mathrm{C} / \mathrm{min}$. The sample was held at -60 ${ }^{\circ} \mathrm{C}$ for $10 \mathrm{~min}$. After that, the temperature increased to $80^{\circ} \mathrm{C}$ at a rate of $5^{\circ} \mathrm{C} / \mathrm{min}$. The melting temperatures were calculated using the heating thermogram. The SFC was calculated at various temperatures by partial integration according to Nassu and Goncalves (1995).

\section{Fatty acid composition by gas chromatography}

The fatty acid composition of butter oil was measured via Agilent 7890A gas chromatograph (Agilent Technologies, Santa Clara, CA, USA) with a split/splitless injector and flame ionization detector HP-88 capillary column $(88 \%$ cianopropylaryl; $100 \mathrm{~m} \times 0.250 \mathrm{~mm}, 0.20 \mu \mathrm{m}$ i.d.). The injector and detector temperatures were 250 and $260^{\circ} \mathrm{C}$, respectively. The oven temperature programme was adjusted as: held at $120^{\circ} \mathrm{C}$ for 1 min, increased to $175^{\circ} \mathrm{C}$ at $10{ }^{\circ} \mathrm{C} / \mathrm{min}$, held at $175^{\circ} \mathrm{C}$ for $10 \mathrm{~min}$, increased to $210^{\circ} \mathrm{C}$ at $5^{\circ} \mathrm{C} / \mathrm{min}$, held at $210^{\circ} \mathrm{C}$ for $5 \mathrm{~min}$, increased to $230^{\circ} \mathrm{C}$ at $5^{\circ} \mathrm{C} / \mathrm{min}$ and held $5 \mathrm{~min}$ at $230^{\circ} \mathrm{C}$. Helium was the carrier gas and its flow rate was $1.5 \mathrm{~mL} / \mathrm{min}$ (Çavdar et al., 2017).

\section{Statistical analysis}

SPSS software, version 22 (IBM, New York, USA) was used for the statistical analysis. A Shapiro-Wilk test was carried out to verify the normality of distribution of data sets. One-way ANOVA analysis followed by Tukey's Post Hoc test $(P<0.05)$ was performed for normally distributed data and Kruskal-Wallis's test was carried out for variables without a normal distribution $(P<0.05)$. All experiments for each sample were triplicated.

\section{RESULTS AND DISCUSSION Moisture content}

The moisture content of butters varied between $12.96-39.69 \%(\mathrm{~m} / \mathrm{m})$ and the mean value is $20.67 \%(\mathrm{~m} / \mathrm{m})$. Turkish national standard (Anonymous, 2021b) specified the maximum moisture content in butter as $16 \%(\mathrm{~m} / \mathrm{m})$. Significant differences were seen among the samples $(P<0.05)$. As seen from Table 1 , seven of 18 butters from national markets and only one of 5 butters from local markets showed suitable moisture content according to the proposed level. All butters from bazaars showed higher moisture content than the accepted moisture content level. Similarly, Akgül et al. (2021) reported 23 of 30 butters obtained from Trabzon were not in accordance with the standard. The wide range in moisture content may result from the differences in production methods, season, type of butter and storage conditions (Akgül et al., 2021). However, high moisture predisposes lipase activity, stimulates microorganisms' growth and hydrolysis of the triglycerides (Idoui et al., 2013).

\section{Fat content}

Milk fat is the main component of butter and therefore, fat analysis is important to evaluate the compositional properties of butter. The fat content of the investigated butters varied between $57.74-84.17 \%(\mathrm{~m} / \mathrm{m})$ (Table1) and the mean value is $74.53 \%(\mathrm{~m} / \mathrm{m})$. Turkish national standard (Anonymous, 2021b) specified the minimum fat content in whole-fat butter as $80 \%(\mathrm{~m} / \mathrm{m})$. The fat content of 7 of $28(25 \%)$ samples complied with the minimum limit for whole-fat butter reported in standards. Interestingly, any sample from bazaar and 16 of 23 samples from the national or local market was not in accordance with the legislation. The legislation reported that "if the milk fat ratio is outside the ranges given in the butter type table, butter is included in the closest subtype." In this case, 20 samples should be classified as half-fat or reduced-fat butter type, although they are not labeled as or sold as half-fat or reduced-fat. Similar results were analyzed by Çakmakçı et al. (2020). They reported that this situation would cause honest producers to suffer financially, and consumers will be malnourished, deceived, and overpaid compared to butter containing more milk fat. Fındık and Andiç (2017) reported the milk fat content of the butters obtained from markets in Van market in the range of $77-83 \%(\mathrm{~m} / \mathrm{m})$. Akgül et al. (2021) reported the fat content of butters obtained from Trabzon was between 41 and $85 \%(\mathrm{~m} / \mathrm{m})$ and 13 of 30 butters were not in accordance with the legislation. 
Table 1 Some physicochemical properties of butters

\begin{tabular}{|c|c|c|c|c|c|c|}
\hline & & $\begin{array}{l}\text { Moisture } \\
(\%, \mathrm{~m} / \mathrm{m})\end{array}$ & $\begin{array}{c}\text { Fat } \\
(\%, \mathrm{~m} / \mathrm{m})\end{array}$ & $\begin{array}{c}\text { NSF } \\
(\%, \mathrm{~m} / \mathrm{m})\end{array}$ & $\begin{array}{c}\text { Salt } \\
(\%, \mathrm{~m} / \mathrm{m})\end{array}$ & $\begin{array}{c}\mathrm{AV} \\
\text { (mg KOH/g fat) }\end{array}$ \\
\hline 1 & \multirow{18}{*}{$\begin{array}{l}\text { National } \\
\text { Markets }\end{array}$} & $16.69 \pm 0.30^{\mathrm{a}, \mathrm{b}, \mathrm{c}}$ & $80.74 \pm 1.20^{\mathrm{fg}, \mathrm{g}, \mathrm{h}, \mathrm{j}, \mathrm{j}}$ & $2.57 \pm 0.04^{\mathrm{a}, \mathrm{b}, \mathrm{c}, \mathrm{d}}$ & $0.13 \pm 0.15^{\mathrm{a}, \mathrm{b}, \mathrm{c}}$ & $4.11 \pm 0.11 \mathrm{a}, \mathrm{b}, \mathrm{c}$ \\
\hline 2 & & $12.96 \pm 0.90^{a}$ & $84.17 \pm 0.10 \mathrm{i}$ & $1.88 \pm 0.52^{\mathrm{a}, \mathrm{b}}$ & $0.14 \pm 0.01^{\mathrm{a}, \mathrm{b}, \mathrm{c}, \mathrm{d}}$ & $3.54 \pm 0.07 \mathrm{a}, \mathrm{b}, \mathrm{c}$ \\
\hline 3 & & $21.69 \pm 1.27 \mathrm{c}$ & $74.32 \pm 1.05^{\mathrm{c}, \mathrm{d}, \mathrm{e}, \mathrm{f}, \mathrm{g}, \mathrm{h}, \mathrm{i}}$ & $3.99 \pm 0.01 \mathrm{a}, \mathrm{b}, \mathrm{c}, \mathrm{d}$ & $0.17 \pm 0.02^{\mathrm{a}, \mathrm{b}, \mathrm{c}, \mathrm{d}}$ & $4.51 \pm 0.37 \mathrm{~b}, \mathrm{c}$ \\
\hline 4 & & $32.63 \pm 0.26^{\mathrm{d}}$ & $62.82 \pm 0.21^{\mathrm{a}, \mathrm{b}}$ & $4.48 \pm 0.32^{b, c}$ & $0.17 \pm 0.00^{\mathrm{a}, \mathrm{b}, \mathrm{c}, \mathrm{d}}$ & $4.93 \pm 0.03^{\mathrm{d}}$ \\
\hline 5 & & $17.02 \pm 1.32^{\mathrm{a}, \mathrm{b}, \mathrm{c}}$ & $73.97 \pm 0.48^{\mathrm{c}, \mathrm{d}, \mathrm{e}, \mathrm{f}, \mathrm{g}, \mathrm{h}}$ & $8.78 \pm 1.00^{\mathrm{e}}$ & $0.14 \pm 0.01^{\mathrm{a}, \mathrm{b}, \mathrm{c}, \mathrm{d}}$ & $3.80 \pm 0.01 \mathrm{a}, \mathrm{b}, \mathrm{c}$ \\
\hline 6 & & $33.20 \pm 1.29 \mathrm{e}$ & $62.63 \pm 1.15^{\mathrm{a}, \mathrm{b}}$ & $4.15 \pm 0.19^{\mathrm{a}, \mathrm{b}, \mathrm{c}, \mathrm{d}}$ & $0.15 \pm 0.00^{\mathrm{a}, \mathrm{b}, \mathrm{c}, \mathrm{d}}$ & $4.60 \pm 0.17 \mathrm{e}$ \\
\hline 7 & & $20.65 \pm 0.27 \mathrm{~b}, \mathrm{c}$ & $75.51 \pm 1.32^{\mathrm{d}, \mathrm{e}, \mathrm{f}, \mathrm{g}, \mathrm{h}, \mathrm{i}}$ & $3.57 \pm 0.58^{\mathrm{a}, \mathrm{b}, \mathrm{c}, \mathrm{d}}$ & $0.17 \pm 0.00^{\mathrm{a}, \mathrm{b}, \mathrm{c}, \mathrm{d}}$ & $3.11 \pm 0.28 \mathrm{a}, \mathrm{b}, \mathrm{c}$ \\
\hline 8 & & $39.69 \pm 0.07^{f}$ & $57.74 \pm 1.46^{a}$ & $1.78 \pm 0.17 \mathrm{a}$ & $0.18 \pm 0.00^{\mathrm{a}, \mathrm{b}, \mathrm{d}}$ & $3.49 \pm 0.49 \mathrm{a}, \mathrm{b}, \mathrm{c}$ \\
\hline 9 & & $25.02 \pm 0.02 \mathrm{~g}$ & $68.20 \pm 2.78^{b, c, d}$ & $5.82 \pm 0.36^{\mathrm{f}}$ & $0.56 \pm 0.01 \mathrm{e}$ & $4.89 \pm 0.17^{f}$ \\
\hline 10 & & $14.38 \pm 0.70^{a, b, c}$ & $75.72 \pm 0.10^{\mathrm{d}, \mathrm{e}, \mathrm{f}, \mathrm{g}, \mathrm{h}, \mathrm{i}}$ & $9.32 \pm 1.00 \mathrm{~g}$ & $0.23 \pm 0.02^{\mathrm{f}}$ & $4.35 \pm 0.06^{\mathrm{b}, \mathrm{c}}$ \\
\hline 11 & & $19.94 \pm 1.32^{\mathrm{a}, \mathrm{b}, \mathrm{c}}$ & $77.20 \pm 1.16^{\mathrm{e}, \mathrm{f}, \mathrm{g}, \mathrm{h}, \mathrm{ij}}$ & $2.49 \pm 0.26^{\mathrm{a}, \mathrm{b}, \mathrm{c}, \mathrm{d}}$ & $0.18 \pm 0.01^{\mathrm{a}, \mathrm{b}, \mathrm{c}, \mathrm{d}}$ & $2.22 \pm 0.24^{a, b, c}$ \\
\hline 12 & & $14.64 \pm 0.01^{\mathrm{a}, \mathrm{b}, \mathrm{c}}$ & $82.27 \pm 1.25^{\mathrm{h}, \mathrm{i}, \mathrm{j}}$ & $2.50 \pm 0.18^{\mathrm{a}, \mathrm{b}, \mathrm{c}, \mathrm{d}}$ & $0.19 \pm 0.00^{\mathrm{b}, \mathrm{c}, \mathrm{d}}$ & $2.99 \pm 0.61^{\mathrm{a}, \mathrm{b}, \mathrm{c}}$ \\
\hline 13 & & $14.32 \pm 0.08^{\mathrm{a}, \mathrm{b}}$ & $82.53 \pm 1.61 \mathrm{i}, \mathrm{j}$ & $2.42 \pm 0.57 \mathrm{a}, \mathrm{b}, \mathrm{c}, \mathrm{d}$ & $0.24 \pm 0.01 \mathrm{~g}$ & $3.96 \pm 0.71^{\mathrm{a}, \mathrm{b}, \mathrm{c}}$ \\
\hline 14 & & $15.15 \pm 0.03 \mathrm{a}, \mathrm{b}, \mathrm{c}$ & $81.79 \pm 1.00^{\mathrm{h}, \mathrm{i}, \mathrm{j}}$ & $2.25 \pm 0.26^{a, b, c, d}$ & $0.19 \pm 0.01 \mathrm{c,d}$ & $2.18 \pm 0.09 \mathrm{a}, \mathrm{b}$ \\
\hline 15 & & $16.61 \pm 0.25^{a, b, c}$ & $80.27 \pm 1.12^{\mathrm{f}, \mathrm{g}, \mathrm{h}, \mathrm{i}, \mathrm{j}}$ & $2.34 \pm 0.32^{\mathrm{a}, \mathrm{b}, \mathrm{c}, \mathrm{d}}$ & $0.21 \pm 0.01^{\mathrm{h}}$ & $2.96 \pm 0.11^{\mathrm{a}, \mathrm{b}, \mathrm{c}}$ \\
\hline 16 & & $16.60 \pm 0.01 \mathrm{a}, \mathrm{b}, \mathrm{c}$ & $77.90 \pm 1.23 \mathrm{e}, \mathrm{fg}, \mathrm{h}, \mathrm{i}, \mathrm{j}$ & $4.86 \pm 0.15^{\mathrm{c}, \mathrm{d}}$ & $0.18 \pm 0.00^{\mathrm{a}, \mathrm{b}, \mathrm{c}, \mathrm{d}}$ & $2.17 \pm 0.10^{a, b, c}$ \\
\hline 17 & & $15.11 \pm 3.33^{\mathrm{a}, \mathrm{b}, \mathrm{c}}$ & $72.76 \pm 1.05^{c, d, e, f}$ & $11.76 \pm 1.00^{\mathrm{h}}$ & $0.16 \pm 0.01 \mathrm{a}, \mathrm{b}, \mathrm{c}, \mathrm{d}$ & $1.99 \pm 0.09 \mathrm{a}$ \\
\hline 18 & & $15.95 \pm 0.21^{\mathrm{a}, \mathrm{b}, \mathrm{c}}$ & $77.25 \pm 1.61 \mathrm{e}, \mathrm{f}, \mathrm{g}, \mathrm{h}, \mathrm{i}, \mathrm{j}$ & $6.02 \pm 1.61^{\mathrm{i}}$ & $0.20 \pm 0.00^{\mathrm{b}}$ & $2.69 \pm 0.01 \mathrm{a,b}, \mathrm{c}$ \\
\hline 19 & \multirow{5}{*}{ Local Markets } & $22.38 \pm 0.01^{\mathrm{c}}$ & $72.77 \pm 0.17 \mathrm{c}, \mathrm{d}, \mathrm{e}, \mathrm{f}$ & $3.99 \pm 0.17 \mathrm{a}, \mathrm{b}, \mathrm{c}, \mathrm{d}$ & $0.16 \pm 0.01 \mathrm{a}, \mathrm{b}, \mathrm{c}, \mathrm{d}$ & $4.46 \pm 1.65^{a, b, c}$ \\
\hline 20 & & $20.30 \pm 0.15^{b, c}$ & $76.80 \pm 0.13^{e, f, g, h, i, j}$ & $2.90 \pm 0.13^{a, b, c, d}$ & $0.11 \pm 0.01^{\mathrm{a}}$ & $5.05 \pm 0.04 \mathrm{~g}$ \\
\hline 21 & & $19.01 \pm 0.53 \mathrm{a} a \mathrm{~b}, \mathrm{c}$ & $78.75 \pm 0.10^{\mathrm{f}, \mathrm{g}, \mathrm{h}, \mathrm{i}, \mathrm{j}}$ & $2.24 \pm 0.10^{\mathrm{a}, \mathrm{b}, \mathrm{c}}$ & $0.15 \pm 0.01 \mathrm{a,c,d}$ & $3.97 \pm 0.31^{\mathrm{a}, \mathrm{b}, \mathrm{c}}$ \\
\hline 22 & & $16.01 \pm 0.80^{a, b, c}$ & $81.32 \pm 0.08 \mathrm{~g}, \mathrm{~h}, \mathrm{i}, \mathrm{j}$ & $2.68 \pm 0.08^{a, b, c, d}$ & $0.12 \pm 0.01 \mathrm{a}, \mathrm{b}$ & $6.09 \pm 1.74^{\mathrm{h}}$ \\
\hline 23 & & $18.50 \pm 1.22^{\mathrm{a}, \mathrm{b}, \mathrm{c}}$ & $77.80 \pm 0.12^{e, f, g, h, i, j}$ & $3.65 \pm 0.12^{\mathrm{a}, \mathrm{b}, \mathrm{c}, \mathrm{d}}$ & $0.14 \pm 0.02^{\mathrm{a}, \mathrm{b}, \mathrm{c}, \mathrm{d}}$ & $6.05 \pm 0.30^{\mathrm{i}}$ \\
\hline 24 & \multirow{5}{*}{$\begin{array}{c}\text { Local } \\
\text { Bazaars }\end{array}$} & $30.90 \pm 0.45^{\mathrm{h}}$ & $67.19 \pm 0.00^{b, c}$ & $2.32 \pm 0.00^{a, b, c, d}$ & $0.18 \pm 0.01 \mathrm{a}, \mathrm{b}, \mathrm{c}, \mathrm{d}$ & $5.42 \pm 0.00$ \\
\hline 25 & & $20.37 \pm 0.40^{\mathrm{b}, \mathrm{c}}$ & $72.79 \pm 1.06^{\mathrm{c}, \mathrm{d}, \mathrm{e}, \mathrm{f}}$ & $5.71 \pm 1.06^{\mathrm{b}}$ & $0.13 \pm 0.01 \mathrm{a}, \mathrm{b}, \mathrm{c}, \mathrm{d}$ & $2.99 \pm 0.27 \mathrm{a}, \mathrm{b}, \mathrm{c}$ \\
\hline 26 & & $20.37 \pm 0.04 \mathrm{~b}, \mathrm{c}$ & $73.00 \pm 0.42^{c, d, e, f, g}$ & $6.98 \pm 0.96 j$ & $0.17 \pm 0.01 \mathrm{a}, \mathrm{b}, \mathrm{c}, \mathrm{d}$ & $3.38 \pm 0.33 \mathrm{a}, \mathrm{b}, \mathrm{c}$ \\
\hline 27 & & $22.90 \pm 0.69^{\mathrm{i}}$ & $70.25 \pm 0.75^{\mathrm{b}, \mathrm{c}, \mathrm{d}, \mathrm{e}}$ & $7.00 \pm 0.42^{\mathrm{k}}$ & $0.28 \pm 0.01^{\mathrm{i}}$ & $5.70 \pm 0.35^{\mathrm{k}}$ \\
\hline 28 & & $25.90 \pm 0.89 i$ & $68.44 \pm 0.34 \mathrm{~b}, \mathrm{c}, \mathrm{d}$ & $4.62 \pm 0.75^{\mathrm{b}, \mathrm{c}, \mathrm{d}}$ & $0.47 \pm 0.01 i$ & $7.60 \pm 0.48^{1}$ \\
\hline Mean & & 20.67 & 74.53 & 4.39 & 0.19 & 4.04 \\
\hline
\end{tabular}

Values are expressed as mean \pm standard deviation. $*$ Different letters in a coloumn (ie within the same category) are significantly different $(P<0.05)$. 
Table 1 Some physicochemical properties of butters (continued)

\begin{tabular}{|c|c|c|c|c|c|c|}
\hline & & $\begin{array}{c}\mathrm{LA} \\
(\%, \mathrm{~m} / \mathrm{m}) \\
\end{array}$ & $\begin{array}{c}\mathrm{OA} \\
(\%, \mathrm{~m} / \mathrm{m})\end{array}$ & $\begin{array}{c}\mathrm{PV} \\
(\mathrm{meq} \mathrm{O} / \mathrm{kg})\end{array}$ & $\begin{array}{c}\text { TBA } \\
\text { (mg MA/kg fat) }\end{array}$ & $\begin{array}{c}\text { LA } \\
(\%, \mathrm{~m} / \mathrm{m})\end{array}$ \\
\hline 1 & \multirow{18}{*}{$\begin{array}{l}\text { National } \\
\text { Markets }\end{array}$} & $0.66 \pm 0.20^{\mathrm{a}, \mathrm{b}, \mathrm{c}, \mathrm{d}}$ & $1.71 \pm 0.05^{\mathrm{a}, \mathrm{b}, \mathrm{c}, \mathrm{d}, \mathrm{e}}$ & $3.26 \pm 0.18^{\mathrm{a}, \mathrm{b}, \mathrm{c}}$ & $0.08 \pm 0.00^{\mathrm{d}, \mathrm{e}, \mathrm{f}, \mathrm{g}, \mathrm{h}}$ & $0.66 \pm 0.20^{\mathrm{a}, \mathrm{b}, \mathrm{c}, \mathrm{d}}$ \\
\hline 2 & & $0.57 \pm 0.01^{\mathrm{a}, \mathrm{b}, \mathrm{c}, \mathrm{d}}$ & $1.54 \pm 0.03^{\mathrm{a}, \mathrm{b}, \mathrm{c}, \mathrm{d}}$ & $0.80 \pm 0.72^{a, b, c}$ & $0.12 \pm 0.00^{\mathrm{h}, \mathrm{i}, \mathrm{j}, \mathrm{k}, \mathrm{l}}$ & $0.57 \pm 0.01^{\mathrm{a}, \mathrm{b}, \mathrm{c}, \mathrm{d}}$ \\
\hline 3 & & $0.72 \pm 0.06^{\mathrm{a}, \mathrm{b}, \mathrm{c}, \mathrm{d}}$ & $1.76 \pm 0.14 \mathrm{a}, \mathrm{b}, \mathrm{c}, \mathrm{d}, \mathrm{e}$ & $2.45 \pm 0.22^{\mathrm{a}, \mathrm{b}, \mathrm{c}, \mathrm{d}}$ & $0.11 \pm 0.00$ h,i,j,k,1 & $0.72 \pm 0.06^{\mathrm{a}, \mathrm{b}, \mathrm{c}, \mathrm{d}}$ \\
\hline 4 & & $0.79 \pm 0.00^{a, b, c, d, e}$ & $1.66 \pm 0.01^{\mathrm{a}, \mathrm{b}, \mathrm{c}, \mathrm{d}, \mathrm{e}}$ & $1.32 \pm 0.60^{a, b, c}$ & $0.14 \pm 0.00^{1}$ & $0.79 \pm 0.00^{\mathrm{a}, \mathrm{b}, \mathrm{c}, \mathrm{d}, \mathrm{e}}$ \\
\hline 5 & & $0.61 \pm 0.03^{\mathrm{a}, \mathrm{b}, \mathrm{c}, \mathrm{d}}$ & $1.57 \pm 0.005^{\mathrm{a}, \mathrm{b}, \mathrm{c}, \mathrm{d}}$ & $3.86 \pm 0.19 \mathrm{a}, \mathrm{b}$ & $0.15 \pm 0.00^{\mathrm{k}, 1}$ & $0.61 \pm 0.03^{\mathrm{a}, \mathrm{b}, \mathrm{c}, \mathrm{d}}$ \\
\hline 6 & & $0.74 \pm 0.04^{\mathrm{a}, \mathrm{b}, \mathrm{c}, \mathrm{d}}$ & $1.53 \pm 0.06^{\mathrm{a}, \mathrm{b}, \mathrm{c}, \mathrm{d}}$ & $7.95 \pm 0.74^{\mathrm{a}, \mathrm{b}, \mathrm{c}}$ & $0.18 \pm 0.00^{\mathrm{m}}$ & $0.74 \pm 0.04^{\mathrm{a}, \mathrm{b}, \mathrm{c}, \mathrm{d}}$ \\
\hline 7 & & $0.50 \pm 0.04^{\mathrm{a}, \mathrm{b}, \mathrm{c}, \mathrm{d}}$ & $1.23 \pm 0.11^{\mathrm{a}, \mathrm{b}}$ & $0.00 \pm 0.79^{\mathrm{a}, \mathrm{b}}$ & $0.10 \pm 0.02^{\mathrm{i}, \mathrm{j}, \mathrm{k}, 1}$ & $0.50 \pm 0.04^{a, b, c, d}$ \\
\hline 8 & & $0.56 \pm 0.08^{\mathrm{a}, \mathrm{b}, \mathrm{c}, \mathrm{d}}$ & $1.05 \pm 0.15^{\mathrm{a}, \mathrm{b}}$ & $4.99 \pm 0.08^{\mathrm{a}, \mathrm{b}, \mathrm{c}}$ & $0.13 \pm 0.01 \mathrm{~d}, \mathrm{e}, \mathrm{f}, \mathrm{g}, \mathrm{h}, \mathrm{i}$ & $0.56 \pm 0.08^{\mathrm{a}, \mathrm{b}, \mathrm{c}, \mathrm{d}}$ \\
\hline 9 & & $0.79 \pm 0.02^{\mathrm{a}, \mathrm{b}, \mathrm{c}, \mathrm{d}, \mathrm{e}}$ & $1.76 \pm 0.06^{\mathrm{a}, \mathrm{b}, \mathrm{c}, \mathrm{d}, \mathrm{e}}$ & $0.69 \pm 0.13^{\mathrm{a}, \mathrm{b}}$ & $0.15 \pm 0.02^{\mathrm{k}, 1}$ & $0.79 \pm 0.02^{\mathrm{a}, \mathrm{b}, \mathrm{c}, \mathrm{d}, \mathrm{e}}$ \\
\hline 10 & & $0.70 \pm 0.01^{\mathrm{a}, \mathrm{b}, \mathrm{c}, \mathrm{d}}$ & $1.49 \pm 0.02^{\mathrm{a}, \mathrm{b}, \mathrm{c}, \mathrm{d}}$ & $7.56 \pm 0.25^{\mathrm{a}, \mathrm{b}, \mathrm{c}, \mathrm{d}, \mathrm{e}}$ & $0.07 \pm 0.01 \mathrm{c}, \mathrm{d}, \mathrm{e}, \mathrm{f}, \mathrm{g}$ & $0.70 \pm 0.01^{\mathrm{a}, \mathrm{b}, \mathrm{c}, \mathrm{d}}$ \\
\hline 11 & & $0.36 \pm 0.04^{\mathrm{a}, \mathrm{b}}$ & $0.89 \pm 0.09^{a}$ & $6.25 \pm 0.00^{\mathrm{f}}$ & $0.04 \pm 0.00^{\mathrm{a}, \mathrm{b}}$ & $0.36 \pm 0.04^{\mathrm{a}, \mathrm{b}}$ \\
\hline 12 & & $0.48 \pm 0.01^{\mathrm{a}, \mathrm{b}, \mathrm{c}}$ & $1.27 \pm 0.26^{\mathrm{a}, \mathrm{b}}$ & $3.73 \pm 1.10^{\mathrm{a}}$ & $0.02 \pm 0.00^{\mathrm{a}, \mathrm{b}, \mathrm{c}, \mathrm{d}}$ & $0.48 \pm 0.01^{\mathrm{a}, \mathrm{b}, \mathrm{c}}$ \\
\hline 13 & & $0.64 \pm 0.04^{a, b, c, d}$ & $1.69 \pm 0.30^{\mathrm{a}, \mathrm{b}, \mathrm{c}, \mathrm{d}, \mathrm{e}}$ & $5.85 \pm 1.03^{\mathrm{a}, \mathrm{b}, \mathrm{c}, \mathrm{d}, \mathrm{e}}$ & $0.06 \pm 0.00^{\mathrm{e}, \mathrm{f}, \mathrm{g}, \mathrm{h}, \mathrm{i}}$ & $0.64 \pm 0.04^{\mathrm{a}, \mathrm{b}, \mathrm{c}, \mathrm{d}}$ \\
\hline 14 & & $0.35 \pm 0.10^{\mathrm{a}, \mathrm{b}}$ & $0.92 \pm 0.04^{\mathrm{a}}$ & $0.60 \pm 0.04^{\mathrm{a}, \mathrm{b}, \mathrm{c}, \mathrm{d}, \mathrm{e}}$ & $0.05 \pm 0.00^{\mathrm{a}, \mathrm{b}, \mathrm{c}}$ & $0.35 \pm 0.10^{\mathrm{a}, \mathrm{b}}$ \\
\hline 15 & & $0.48 \pm 0.11 \mathrm{a}, \mathrm{b}, \mathrm{c}$ & $1.23 \pm 0.04 \mathrm{a}, \mathrm{b}$ & $1.98 \pm 0.41^{\mathrm{a}, \mathrm{b}}$ & $0.10 \pm 0.00^{\mathrm{f}, \mathrm{g}, \mathrm{h}, \mathrm{i}, \mathrm{j}}$ & $0.48 \pm 0.11^{\mathrm{a}, \mathrm{b}, \mathrm{c}}$ \\
\hline 16 & & $0.35 \pm 0.01^{\mathrm{a}, \mathrm{b}}$ & $0.90 \pm 0.00^{\mathrm{a}}$ & $3.86 \pm 0.28^{\mathrm{d}, \mathrm{e}}$ & $0.04 \pm 0.00^{\mathrm{a}, \mathrm{b}, \mathrm{c}, \mathrm{d}, \mathrm{e}}$ & $0.35 \pm 0.01^{\mathrm{a}, \mathrm{b}}$ \\
\hline 17 & & $0.32 \pm 0.00^{a}$ & $0.84 \pm 0.04^{\mathrm{a}}$ & $0.94 \pm 0.57 \mathrm{a}, \mathrm{b}, \mathrm{c}$ & $0.11 \pm 0.00 \mathrm{~g}, \mathrm{~h}, \mathrm{i}, \mathrm{j}$ & $0.32 \pm 0.00^{\mathrm{a}}$ \\
\hline 18 & & $0.43 \pm 0.015^{\mathrm{a}, \mathrm{b}, \mathrm{c}}$ & $1.13 \pm 0.00^{\mathrm{a}, \mathrm{b}}$ & $3.59 \pm 0.57 \mathrm{c}, \mathrm{d}, \mathrm{e}$ & $0.01 \pm 0.00^{\mathrm{a}}$ & $0.43 \pm 0.015^{\mathrm{a}, \mathrm{b}, \mathrm{c}}$ \\
\hline 19 & \multirow{5}{*}{$\begin{array}{c}\text { Local } \\
\text { Markets }\end{array}$} & $0.72 \pm 0.00^{\mathrm{a}, \mathrm{b}, \mathrm{c}, \mathrm{d}}$ & $1.73 \pm 0.64^{\mathrm{a}, \mathrm{b}, \mathrm{c}, \mathrm{d}, \mathrm{e}}$ & $6.02 \pm 2.24^{\mathrm{c}, \mathrm{d}, \mathrm{e}}$ & $0.16 \pm 0.00$ e,f,g,h,i & $0.72 \pm 0.00^{\mathrm{a}, \mathrm{b}, \mathrm{c}, \mathrm{d}}$ \\
\hline 20 & & $0.81 \pm 0.26^{\mathrm{b}, \mathrm{c}, \mathrm{d}, \mathrm{e}}$ & $2.01 \pm 0.01$ a,b,c,d,e & $2.75 \pm 0.57 \mathrm{a}, \mathrm{b}, \mathrm{c}$ & $0.15 \pm 0.00^{\mathrm{k}, 1}$ & $0.81 \pm 0.26^{\mathrm{b}, \mathrm{c}, \mathrm{d}, \mathrm{e}}$ \\
\hline 21 & & $0.64 \pm 0.01^{\mathrm{a}, \mathrm{b}, \mathrm{c}, \mathrm{d}}$ & $1.11 \pm 0.08^{\mathrm{a}, \mathrm{b}}$ & $3.78 \pm 1.86^{\mathrm{c}, \mathrm{d}, \mathrm{e}}$ & $0.10 \pm 0.00^{\mathrm{f}, \mathrm{g}, \mathrm{h}, \mathrm{i}, \mathrm{j}}$ & $0.64 \pm 0.01^{\mathrm{a}, \mathrm{b}, \mathrm{c}, \mathrm{d}}$ \\
\hline 22 & & $0.98 \pm 0.05^{\mathrm{d}, \mathrm{e}}$ & $2.55 \pm 0.73^{\mathrm{d}, \mathrm{e}}$ & $1.00 \pm 0.59^{\mathrm{a}, \mathrm{b}}$ & $0.08 \pm 0.02^{\mathrm{e}, \mathrm{f}, \mathrm{g}, \mathrm{h}, \mathrm{i}}$ & $0.98 \pm 0.05^{\mathrm{d}, \mathrm{e}}$ \\
\hline 23 & & $0.97 \pm 0.28 \mathrm{~d}, \mathrm{e}$ & $2.46 \pm 0.12^{\mathrm{c}, \mathrm{d}, \mathrm{e}}$ & $2.98 \pm 0.39 \mathrm{a}, \mathrm{b}, \mathrm{c}$ & $0.13 \pm 0.01^{\mathrm{j}, \mathrm{k}, 1}$ & $0.97 \pm 0.28 \mathrm{~d}, \mathrm{e}$ \\
\hline 24 & \multirow{5}{*}{$\begin{array}{c}\text { Local } \\
\text { Bazaars }\end{array}$} & $0.87 \pm 0.05^{\mathrm{c}, \mathrm{d}, \mathrm{e}}$ & $1.67 \pm 0.00^{\mathrm{a}, \mathrm{b}, \mathrm{c}, \mathrm{d}, \mathrm{e}}$ & $2.98 \pm 0.97 \mathrm{a}, \mathrm{b}, \mathrm{c}, \mathrm{d}, \mathrm{e}$ & $0.08 \pm 0.01$ e,f,g,h,i & $0.87 \pm 0.05^{\mathrm{c}, \mathrm{d}, \mathrm{e}}$ \\
\hline 25 & & $0.48 \pm 0.45^{\mathrm{a}, \mathrm{b}, \mathrm{c}}$ & $1.23 \pm 0.11^{\mathrm{a}, \mathrm{b}}$ & $4.48 \pm 0.93^{\mathrm{a}, \mathrm{b}}$ & $0.08 \pm 0.00$ ef,g,h,i & $0.48 \pm 0.45^{\mathrm{a}, \mathrm{b}, \mathrm{c}}$ \\
\hline 26 & & $0.54 \pm 0.00^{\mathrm{a}, \mathrm{b}, \mathrm{c}, \mathrm{d}}$ & $1.34 \pm 0.13^{\mathrm{a}, \mathrm{b}, \mathrm{c}}$ & $3.24 \pm 0.20^{\mathrm{a}, \mathrm{b}}$ & $0.24 \pm 0.00^{\mathrm{m}}$ & $0.54 \pm 0.00^{\mathrm{a}, \mathrm{b}, \mathrm{c}, \mathrm{d}}$ \\
\hline 27 & & $0.91 \pm 0.08^{\mathrm{c}, \mathrm{d}, \mathrm{e}}$ & $2.19 \pm 0.015^{\mathrm{b}, \mathrm{c}, \mathrm{d}, \mathrm{e}}$ & $0.59 \pm 0.40^{a, b, c, d}$ & $0.06 \pm 0.00^{\mathrm{b}, \mathrm{c}, \mathrm{d}, \mathrm{e}, \mathrm{f}}$ & $0.91 \pm 0.08^{\mathrm{c}, \mathrm{d}, \mathrm{e}}$ \\
\hline 28 & & $1.22 \pm 0.03^{\mathrm{e}}$ & $2.81 \pm 0.18^{\mathrm{e}}$ & $3.56 \pm 0.40^{a, b, c, d}$ & $0.04 \pm 0.00^{\mathrm{a}, \mathrm{b}, \mathrm{c}, \mathrm{d}, \mathrm{e}}$ & $1.22 \pm 0.03^{\mathrm{e}}$ \\
\hline Mean & & 0.65 & 1.55 & 3.25 & 0.09 & 0.65 \\
\hline
\end{tabular}

Values are expressed as mean \pm standard deviation. $*$ Different letters in a coloumn (ie within the same category) are significantly different $(P<0.05)$.

\section{Non-fat solid content}

Non-fat solid content of butters changed between $1.78-11.76 \%(\mathrm{~m} / \mathrm{m})$ and the mean value was $4.39 \%(\mathrm{~m} / \mathrm{m})$. According to the Turkish national standard, the maximum milk non-fat solid content must be $2 \%(\mathrm{~m} / \mathrm{m})$ (Anonymous, 2021). In this manner, 27 of $28(96.42 \%)$ butters showed higher non-fat solid content than the maximum limit in the related standards. Non-fat solid content, including lactose, protein, minerals, etc., enhances aromatic compounds and nutritional value. However, they are not desirable in the butter since they can increase microbial spoilage risk. Moreover, these results showed that dairies 
lacked modern technology and appropriate equipment for churning, washing, and malaxation, directly related to non-fat solid content as mentioned by Çelik and Bakirci (2000). Dervişoğlu et al. (2013) noted that 59 of 88 $(67.05 \%)$ butters supplied from the Black Sea region did not comply with non-fat solids content. Altun et al. (2011) reported that non-fat solid content of $90 \%$ of the butters and fat content of $70 \%$ of the butters obtained from market in Van was out of the range suggested by Turkish national standard.

\section{Salt content}

Salt is used to inhibit the growth of bacteria and microorganisms and is a natural preservative to extend the shelf-life of food products on the market. On the other hand, high salt consumption causes health issues such as hypertension (Haron et al., 2020). The salt content of investigated butters ranged from 0.11 to $0.56 \%(\mathrm{~m} / \mathrm{m})$, and the average value was found as $0.19 \%(\mathrm{~m} / \mathrm{m})$. According to the Turkish national standard, the maximum salt content must be $2 \%$ (Anonymous, 2021b). Thus, all the salt content of the butters complied with the proposed values in legislation. Similarly, Akgül et al. (2021) found that all butters supplied from Trabzon were coherent with the Turkish standard. Haron et al. (2020) reported that $79.5 \%$ of butter and margarine products in Malaysia had an average salt content above the reference value stated in the International Product Criteria.

\section{Acid value}

The acid value is the amount $\mathrm{KOH}$ in milligram required to neutralize the free fatty acids in one gram of pure oil. It is critical to determine the acid value, which is an indicator of free fatty acid degradation, to evaluate butter's storage stability and quality. The presence of free fatty acids in butter cause flavor degradation (fatty acids with less than $16 \mathrm{C}$ atoms) and carry the risk of creating oxidation reactions (Findık and Andiç, 2017). The minimum acid value for the investigated butters was $1.99 \mathrm{mg} \mathrm{KOH} / \mathrm{g}$ butter oil, while the maximum was $7.60 \mathrm{mg} \mathrm{KOH/g}$ butter oil. Atamer (1993) reported that if the amount of free fatty acids reaches $1.80 \mathrm{mg} \mathrm{KOH} / \mathrm{g}$ fat, a perceptible change occurs in the taste of butter. If it reaches $3.30 \mathrm{mg} \mathrm{KOH} / \mathrm{g}$ fat, a significant bitterness occurs in the butter (Atamer and Sezgin, 1984). According to our results, all butters had an acid value higher than $1.8 \mathrm{mg} \mathrm{KOH} / \mathrm{g}$ fat, which means all butters have a perceptible change in taste. Besides, 4 of 28 samples had an acid value greater than $3.3 \mathrm{mg} \mathrm{KOH} / \mathrm{g}$ fat. Acid values of three butters (B22, B23, B28) were relatively high (over $4 \mathrm{mg} \mathrm{KOH/g}$ oil) and this can cause lipolytic rancidity. The acid value is associated with an excess of free fatty acid due to the hydrolysis of triglycerides in fat and oil (Demirkol et al., 2016). Thus, a higher acid value indicates unfavorable processing and storage circumstances, such as increased storage temperature and relative humidity and increased lipase activity in butter due to insufficient thermal processing (Wilbey, 2009; Demirkol et al., 2016).

\section{Titratable acidity}

Lactic acid of the butters ranged between 0.32 and $0.98 \%(\mathrm{~m} / \mathrm{m})$ and the mean value is $0.49 \%(\mathrm{~m} / \mathrm{m})$ (Table 1). The mean titratable acidity values of the investigated butters were lower than reported values of butter obtained from the Black sea region (mean $=0.55 \%, \mathrm{~m} / \mathrm{m}$ ) (Dervisoglu et al., 2013) and obtained from Trabzon, Turkey (mean $=0.86 \%, \mathrm{~m} / \mathrm{m}$ ) (Akgül et al., 2021). High titratable acidity (\% lactic acid) can be associated with undesirable microflora and the use of lowquality milk for the production of butter (Demirkol et al., 2016). Besides, the higher lactic acid values may be related to lipid degradation, where there is a release of aldehydes or conversion of residual lactose into lactic acid, caused by inadequacy in processing or excessive exposure to light (da Silva et al., 2020). Oleic acid of the butters ranged between 0.89 and $2.81 \%$ $(\mathrm{m} / \mathrm{m})$, and the mean value was $1.55 \%$ (Table 1$)$. The differences in the acidity (\% oleic acid) of butters may be related to the characteristics of the cream and yoghurt from which they are produced, the method the butter is produced and, the number of microorganisms that develop the acidity (Ergin, 1976). Méndez-Cid et al. (2017) reported the acidity of salted and unsalted butter as 0.35 and 0.42 (oleic acid\%), respectively. These researchers reported that acidity increased 2.8 
times higher at the end of the nine-month storage than in fresh butter and higher storage temperatures led to higher acidity values; the presence of salt also produced a slight increase in the values (Méndez-Cid et al., 2017). The maximum limit of acidity is $0.27 \%(\mathrm{~m} / \mathrm{m})$ for whole-fat butters (Anonymous, 2021b). In this manner, all butters had higher acidity values than the recommended value.

\section{Peroxide value}

Peroxide is the primary oxidation product and peroxide value is a commonly used quality parameter for fats and oils. Oxidative rancidity is influenced by many factors such as fatty acid composition, oxygen availability, light and temperature exposure, minor components in the oil/fat (Flakelar et al., 2015). Turkish national standard (Anonymous, 2008; 2015) does not report a maximum limit of peroxide value for salted butter. However, EC Commission regulations (Anonymous, 2008) specified the maximum value of peroxide detected must be below 0.3 meq $\mathrm{O}_{2} / \mathrm{kg}$ fat for unsalted butter. The peroxide value of the investigated butters was between 0.00 and 8.49 meq $\mathrm{O}_{2} / \mathrm{kg}$ fat. If $0.3 \mathrm{meq}$ $\mathrm{O}_{2} / \mathrm{kg}$ fat is taken as reference, 27 of $28(\% 96.42)$ butters had a peroxide value higher than $0.3 \mathrm{meq}$ $\mathrm{O}_{2} / \mathrm{kg}$. These findings indicate that most butters had unsuitable production, storage or transportation conditions. Additionally, Atamer (1993) reported that if the peroxide number in butter reaches 2 meq $\mathrm{O}_{2} / \mathrm{kg}$ fat, an unusually bland taste is perceived. The peroxide value of 19 of $28(67.85 \%)$ butters obtained from market and bazaars was higher than 2. Our results are generally in parallel to the peroxide value of butters reported in the literature (Akgül et al., 2021; Celik, Bakirci, 2000; Sevmiş et al., 2020).

\section{TBA value}

TBA value gives the amount of malonaldehyde at the advanced stages of oxidation. There is not any limitation for TBA values in the European and Turkish legislation of butter (Anonymous 2008; 2015). Atamer et al. (1982) reported that rancid taste is noticeable when TBA values are higher than $0.3 \mathrm{mg}$ malonaldehyde/ $\mathrm{kg}$ fat. TBA value of the investigated butters was between 0.02 and
$0.24 \mathrm{MA} / \mathrm{kg}$ fat, and the mean value was 0.096 $\mathrm{mg} \mathrm{MA} / \mathrm{kg}$ fat. If $0.3 \mathrm{mg} \mathrm{MA} / \mathrm{kg}$ fat is considered as a reference, all-butters have a lower TBA value than $0.3 \mathrm{mg} \mathrm{MA} / \mathrm{kg}$ fat. Demirkaya (2013) noted that TBA value of 50 butters from Bilecik market changed between 0.078-0.236 $\mu \mathrm{g}$ $\mathrm{MA} / \mathrm{g}$ and the average TBA value was $0.145 \pm 0.038 \mu \mathrm{g} \mathrm{MA} / \mathrm{g}$.

\section{Fatty acid composition}

The milk fat composition is affected by several factors such as animal species, nutrition, climate and environmental conditions (Samet-Bali et al., 2009) and it is not constant. Thus, there is not an expected standard fatty acid composition for butters. In the present study, saturated fatty acid (SFA), monounsaturated fatty acid (MUFA) and polyunsaturated fatty acid content (PUFA) varied between $61.87-73.19 \%, 23.14-34.08 \%$ and $1.60-$ $3.59 \%$, respectively. Similarly, Dervişoglu et al. (2013) analyzed the fatty acid composition of 88 butters from the Black sea region. They reported the average percentage composition of SFA, MUFA, and PUFA as 73.63, 23.12, and 1.62, respectively. In our study, the predominant fatty acids of butters were palmitic $(31.09 \%)$, followed by oleic, stearic, and myristic acid with average percentages of 26.07, 12.64 and 10.98, respectively. Akgül et al. (2021) also reported the primary fatty acid composition of butters from Trabzon as palmitic $(35.15 \%)$, oleic (28.13), stearic $(14.19 \%)$, and myristic acid $(10.77 \%)$. Besides, they reported a lower butyric acid content $(0.47 \%)$ compared to our results $(2.41 \%)$. Butyric acid is another important fatty acid of butters. Butyric acid is reported as a potent inhibitor of cancer cell proliferation, whereas it is also responsible for the rancidity of butter resulting from lipolysis (Dervisoglu et al., 2013). Investigated butters contain two essential fatty acids, linoleic and linolenic acids, at the average percentages of 0.35 and 1.27, respectively. B19, $\mathrm{B} 20$ and B21 had higher C10:0 content than other butters (Table 2). C10:0 is a fatty acid widely presented in palm kernel and coconut (Konkol and Rasmussen, 2015). 
Table 2. Fatty acid composition of butters (\%)

\begin{tabular}{|c|c|c|c|c|c|}
\hline Butters & C4:0 & C6:0 & C8:0 & C10:0 & C12:0 \\
\hline 1 & $2.18 \pm 0.39^{\mathrm{b}}$ & $1.71 \pm 0.04^{\mathrm{a}}$ & $1.15 \pm 0.08^{\mathrm{a}}$ & $2.72 \pm 0.29^{a}$ & $3.28 \pm 0.19^{\mathrm{a}}$ \\
\hline 2 & $2.06 \pm 0.41^{\mathrm{b}}$ & $1.68 \pm 0.15^{\mathrm{a}}$ & $1.12 \pm 0.08^{\mathrm{a}}$ & $2.68 \pm 0.05^{\mathrm{a}}$ & $3.19 \pm 0.14^{\mathrm{a}}$ \\
\hline 3 & $2.53 \pm 0.41^{\mathrm{b}}$ & $1.74 \pm 0.01^{\mathrm{a}}$ & $1.11 \pm 0.20^{\mathrm{a}}$ & $2.57 \pm 0.07^{\mathrm{a}}$ & $3.03 \pm 0.25^{\mathrm{a}}$ \\
\hline 4 & $2.53 \pm 0.69^{\mathrm{b}}$ & $1.73 \pm 0.00^{\mathrm{a}}$ & $1.11 \pm 0.29^{\mathrm{a}}$ & $2.64 \pm 0.46^{a}$ & $3.39 \pm 0.38^{\mathrm{a}}$ \\
\hline 5 & $2.56 \pm 0.26^{\mathrm{b}}$ & $1.78 \pm 0.03^{\mathrm{a}}$ & $1.15 \pm 0.14^{\mathrm{a}}$ & $2.67 \pm 0.18^{a}$ & $3.20 \pm 0.48^{\mathrm{a}}$ \\
\hline 6 & $2.37 \pm 0.26^{\mathrm{b}}$ & $1.67 \pm 0.12^{\mathrm{a}}$ & $1.08 \pm 0.11^{\mathrm{a}}$ & $2.58 \pm 0.24^{a}$ & $3.00 \pm 0.16^{\mathrm{a}}$ \\
\hline 7 & $2.56 \pm 0.31^{\mathrm{b}}$ & $1.78 \pm 0.14^{a}$ & $1.14 \pm 0.12^{\mathrm{a}}$ & $2.67 \pm 0.11^{\mathrm{a}}$ & $3.15 \pm 0.21^{\mathrm{a}}$ \\
\hline 8 & $2.37 \pm 0.40^{\mathrm{b}}$ & $1.59 \pm 0.38^{\mathrm{a}}$ & $1.01 \pm 0.01^{\mathrm{a}}$ & $2.32 \pm 0.05^{\mathrm{a}}$ & $2.78 \pm 0.14^{\mathrm{a}}$ \\
\hline 9 & $2.67 \pm 0.41^{\mathrm{b}}$ & $1.80 \pm 0.21^{\mathrm{a}}$ & $1.13 \pm 0.02^{\mathrm{a}}$ & $2.58 \pm 0.31^{a}$ & $3.08 \pm 0.28^{\mathrm{a}}$ \\
\hline 10 & $2.33 \pm 0.84^{b, c}$ & $1.73 \pm 0.24^{\mathrm{a}}$ & $0.00 \pm 0.01^{\mathrm{a}}$ & $2.73 \pm 0.05^{\mathrm{a}}$ & $3.58 \pm 0.39^{\mathrm{a}}$ \\
\hline 11 & $2.58 \pm 0.98^{\mathrm{b}, \mathrm{c}}$ & $1.80 \pm 0.21^{\mathrm{a}}$ & $1.16 \pm 0.18^{\mathrm{a}}$ & $2.75 \pm 0.08^{a}$ & $3.27 \pm 0.11^{\mathrm{a}}$ \\
\hline 12 & $2.56 \pm 0.98^{\mathrm{b}}$ & $1.77 \pm 0.08^{\mathrm{a}}$ & $1.15 \pm 0.91^{\mathrm{a}}$ & $2.71 \pm 0.21^{a}$ & $3.26 \pm 0.35^{\mathrm{a}}$ \\
\hline 13 & $2.62 \pm 0.97 \mathrm{~b}, \mathrm{c}$ & $1.82 \pm 0.04^{\mathrm{a}}$ & $0.00 \pm 0.00^{\mathrm{a}}$ & $2.75 \pm 0.26^{a}$ & $3.28 \pm 0.11^{\mathrm{a}}$ \\
\hline 14 & $2.56 \pm 0.27^{\mathrm{b}}$ & $1.75 \pm 0.07^{\mathrm{a}}$ & $1.13 \pm 0.04^{\mathrm{a}}$ & $2.62 \pm 0.04^{\mathrm{a}}$ & $3.08 \pm 0.16^{\mathrm{a}}$ \\
\hline 15 & $2.61 \pm 0.55^{\mathrm{b}}$ & $1.79 \pm 0.21^{\mathrm{a}}$ & $1.13 \pm 0.02^{\mathrm{a}}$ & $2.62 \pm 0.04^{\mathrm{a}}$ & $3.12 \pm 0.11^{\mathrm{a}}$ \\
\hline 16 & $2.68 \pm 0.26^{\mathrm{b}}$ & $1.79 \pm 0.19^{\mathrm{a}}$ & $1.11 \pm 0.02^{\mathrm{a}}$ & $2.54 \pm 0.25^{\mathrm{a}}$ & $3.06 \pm 0.08^{\mathrm{a}}$ \\
\hline 17 & $2.53 \pm 0.13^{\mathrm{b}}$ & $1.80 \pm 0.25^{\mathrm{a}}$ & $1.17 \pm 0.21^{\mathrm{a}}$ & $2.78 \pm 0.12^{\mathrm{a}}$ & $3.38 \pm 0.29^{\mathrm{a}}$ \\
\hline 18 & $2.52 \pm 0.27^{b, c}$ & $1.86 \pm 0.15^{\mathrm{a}}$ & $1.24 \pm 0.36^{\mathrm{a}}$ & $3.02 \pm 0.05^{\mathrm{a}}$ & $3.70 \pm 0.28^{\mathrm{a}}$ \\
\hline 19 & $2.20 \pm 0.97^{a}$ & $2.20 \pm 0.49^{\mathrm{a}}$ & $0.01 \pm 0.00^{\mathrm{a}}$ & $7.26 \pm 0.55^{\mathrm{a}}$ & $3.03 \pm 0.11^{\mathrm{a}}$ \\
\hline 20 & $1.93 \pm 0.97^{\mathrm{a}}$ & $2.15 \pm 0.28^{\mathrm{a}}$ & $2.49 \pm 0.18^{\mathrm{a}}$ & $8.64 \pm 0.74^{a}$ & $3.69 \pm 0.38^{\mathrm{a}}$ \\
\hline 21 & $1.98 \pm 0.97^{\mathrm{a}}$ & $2.15 \pm 0.28^{\mathrm{a}}$ & $2.44 \pm 0.18^{a}$ & $8.19 \pm 0.14^{a}$ & $3.49 \pm 0.11^{\mathrm{a}}$ \\
\hline 22 & $2.39 \pm 0.13^{\mathrm{b}}$ & $1.63 \pm 0.25^{\mathrm{a}}$ & $1.01 \pm 0.02^{\mathrm{a}}$ & $2.31 \pm 0.45^{\mathrm{a}}$ & $2.74 \pm 0.26^{\mathrm{a}}$ \\
\hline 23 & $2.43 \pm 0.41^{\mathrm{b}}$ & $1.71 \pm 0.08^{\mathrm{a}}$ & $1.09 \pm 0.09^{\mathrm{a}}$ & $2.53 \pm 0.12^{\mathrm{a}}$ & $3.01 \pm 0.18^{\mathrm{a}}$ \\
\hline 24 & $2.24 \pm 0.40^{\mathrm{b}}$ & $1.50 \pm 0.38^{\mathrm{a}}$ & $0.93 \pm 0.05^{\mathrm{a}}$ & $2.10 \pm 0.29^{a}$ & $2.46 \pm 0.29^{\mathrm{a}}$ \\
\hline 25 & $2.26 \pm 0.38^{c}$ & $1.78 \pm 0.73^{\mathrm{a}}$ & $1.23 \pm 0.32^{\mathrm{a}}$ & $3.16 \pm 0.21^{\mathrm{a}}$ & $3.99 \pm 0.15^{\mathrm{a}}$ \\
\hline 26 & $2.37 \pm 0.69^{\mathrm{b}}$ & $1.69 \pm 0.16^{\mathrm{a}}$ & $1.10 \pm 0.21^{\mathrm{a}}$ & $2.53 \pm 0.29^{a}$ & $3.03 \pm 0.25^{\mathrm{a}}$ \\
\hline 27 & $2.28 \pm 0.41^{\mathrm{b}}$ & $1.65 \pm 0.65^{\mathrm{a}}$ & $1.05 \pm 0.02^{\mathrm{a}}$ & $2.50 \pm 0.22^{\mathrm{a}}$ & $3.02 \pm 0.04^{\mathrm{a}}$ \\
\hline 28 & $2.57 \pm 0.41^{\mathrm{b}}$ & $1.42 \pm 0.15^{\mathrm{a}}$ & $0.80 \pm 0.25^{\mathrm{a}}$ & $1.67 \pm 0.15^{\mathrm{a}}$ & $1.90 \pm 0.04^{\mathrm{a}}$ \\
\hline Mean & 2.41 & 1.77 & 1.08 & 3.17 & 3.15 \\
\hline
\end{tabular}

Table 2. (Continued) Fatty acid composition of butters (\%)

\begin{tabular}{|c|c|c|c|c|c|}
\hline Butters & C14:0 & C15:0 & C16:0 & C18:0 & C18:1 \\
\hline 1 & $11.60 \pm 0.52^{\mathrm{a}}$ & $1.27 \pm 0.15^{\mathrm{a}}$ & $32.78 \pm 1.37^{\mathrm{d}}$ & $11.94 \pm 1.18^{\mathrm{a}, \mathrm{b}, \mathrm{c}}$ & $24.56 \pm 0.79^{\mathrm{a}, \mathrm{b}}$ \\
\hline 2 & $11.35 \pm 0.49^{\mathrm{a}}$ & $1.18 \pm 0.09^{\mathrm{a}}$ & $31.08 \pm 0.26^{\mathrm{a}, \mathrm{b}, \mathrm{c}}$ & $12.63 \pm 0.87^{\mathrm{b}, \mathrm{c}}$ & $26.16 \pm 0.24^{\mathrm{a}, \mathrm{b}, \mathrm{c}, \mathrm{d}}$ \\
\hline 3 & $10.97 \pm 1.21^{\mathrm{a}}$ & $1.16 \pm 0.07^{\mathrm{a}}$ & $31.03 \pm 1.31^{\mathrm{a}, \mathrm{b}, \mathrm{c}}$ & $13.05 \pm 0.26^{\mathrm{d}}$ & $26.31 \pm 1.83^{\mathrm{a}, \mathrm{b}, \mathrm{c}, \mathrm{d}, \mathrm{e}}$ \\
\hline 4 & $11.20 \pm 0.31^{\mathrm{a}}$ & $1.20 \pm 0.11^{\mathrm{a}}$ & $30.53 \pm 0.76^{\mathrm{a}, \mathrm{b}, \mathrm{c}}$ & $12.82 \pm 1.15^{\mathrm{b}, \mathrm{c}}$ & $26.17 \pm 1.64^{\mathrm{a}, \mathrm{b}, \mathrm{c}, \mathrm{d}}$ \\
\hline 5 & $11.50 \pm 0.87^{\mathrm{a}}$ & $1.15 \pm 0.18^{\mathrm{a}}$ & $31.17 \pm 0.86^{\mathrm{a}, \mathrm{b}, \mathrm{c}}$ & $12.60 \pm 0.83^{\mathrm{a}, \mathrm{b}, \mathrm{c}}$ & $25.60 \pm 1.55^{\mathrm{a}, \mathrm{b}, \mathrm{c}, \mathrm{d}}$ \\
\hline 6 & $10.95 \pm 1.17^{\mathrm{a}}$ & $1.27 \pm 0.24^{\mathrm{a}}$ & $30.67 \pm 1.11^{\mathrm{a}, \mathrm{b}, \mathrm{c}}$ & $11.96 \pm 1.06^{\mathrm{a}, \mathrm{b}, \mathrm{c}}$ & $27.95 \pm 1.56^{\mathrm{c}, \mathrm{d}, \mathrm{e}}$ \\
\hline 7 & $11.39 \pm 0.72^{\mathrm{a}}$ & $1.21 \pm 0.04^{\mathrm{a}}$ & $31.24 \pm 0.55^{\mathrm{a}, \mathrm{b}, \mathrm{c}}$ & $12.48 \pm 0.98^{\mathrm{a}, \mathrm{b}, \mathrm{c}}$ & $25.70 \pm 1.31^{\mathrm{a}, \mathrm{b}, \mathrm{c}, \mathrm{d}}$ \\
\hline 8 & $10.27 \pm 0.38^{\mathrm{a}}$ & $1.12 \pm 0.02^{\mathrm{a}}$ & $29.26 \pm 0.38^{\mathrm{a}, \mathrm{b}, \mathrm{c}}$ & $13.05 \pm 0.28^{\mathrm{e}}$ & $29.74 \pm 1.35^{\mathrm{d}, \mathrm{e}, \mathrm{f}}$ \\
\hline 9 & $11.07 \pm 0.45^{\mathrm{a}}$ & $1.09 \pm 0.11^{\mathrm{a}}$ & $31.23 \pm 0.69^{\mathrm{a}, \mathrm{b}, \mathrm{c}}$ & $12.57 \pm 0.83^{\mathrm{a}, \mathrm{b}, \mathrm{c}}$ & $26.06 \pm 1.41^{\mathrm{a}, \mathrm{b}, \mathrm{c}, \mathrm{d}}$ \\
\hline 10 & $12.54 \pm 0.94^{\mathrm{a}}$ & $1.61 \pm 0.22^{\mathrm{a}}$ & $38.09 \pm 0.91^{\mathrm{e}}$ & $9.26 \pm 0.42^{\mathrm{a}}$ & $22.43 \pm 1.06^{\mathrm{a}, \mathrm{b}}$ \\
\hline 11 & $11.61 \pm 0.69^{\mathrm{a}}$ & $1.26 \pm 0.08^{\mathrm{a}}$ & $32.59 \pm 0.69^{f}$ & $11.97 \pm 0.55^{\mathrm{a}, \mathrm{b}, \mathrm{c}}$ & $24.58 \pm 0.80^{\mathrm{a}, \mathrm{b}, \mathrm{c}}$ \\
\hline 12 & $11.56 \pm 0.65^{\mathrm{a}}$ & $1.23 \pm 0.08^{\mathrm{a}}$ & $32.45 \pm 0.80^{\mathrm{g}}$ & $12.01 \pm 0.32^{\mathrm{a}, \mathrm{b}, \mathrm{c}}$ & $24.82 \pm 1.48^{\mathrm{a}, \mathrm{b}, \mathrm{c}}$ \\
\hline 13 & $11.67 \pm 0.80^{\mathrm{a}}$ & $1.29 \pm 0.25^{\mathrm{a}}$ & $32.08 \pm 0.74^{\mathrm{c}}$ & $12.48 \pm 0.83^{\mathrm{a}, \mathrm{b}, \mathrm{c}}$ & $25.28 \pm 0.41^{\mathrm{a}, \mathrm{b}, \mathrm{c}, \mathrm{d}}$ \\
\hline 14 & $11.01 \pm 0.77^{\mathrm{a}}$ & $1.21 \pm 0.21^{\mathrm{a}}$ & $29.72 \pm 0.22^{\mathrm{a}, \mathrm{b}, \mathrm{c}}$ & $12.00 \pm 0.31^{\mathrm{a}, \mathrm{b}, \mathrm{c}}$ & $28.63 \pm 1.21^{\mathrm{c}, \mathrm{d}, \mathrm{e}, \mathrm{f}}$ \\
\hline 15 & $11.38 \pm 0.39^{\mathrm{a}}$ & $1.18 \pm 0.00^{\mathrm{a}}$ & $30.83 \pm 1.01^{\mathrm{a}, \mathrm{b}, \mathrm{c}}$ & $12.92 \pm 0.50^{\mathrm{c}}$ & $25.97 \pm 1.23^{\mathrm{a}, \mathrm{b}, \mathrm{c}, \mathrm{d}}$ \\
\hline 16 & $11.40 \pm 0.87^{\mathrm{a}}$ & $1.16 \pm 0.07^{\mathrm{a}}$ & $31.85 \pm 1.85^{\mathrm{b}, \mathrm{c}}$ & $12.73 \pm 1.34^{\mathrm{a}, \mathrm{b}, \mathrm{c}}$ & $25.21 \pm 1.07^{\mathrm{a}, \mathrm{b}, \mathrm{c}, \mathrm{d}}$ \\
\hline 17 & $11.92 \pm 0.50^{\mathrm{a}}$ & $1.22 \pm 0.16^{\mathrm{a}}$ & $32.96 \pm 1.54^{\mathrm{h}}$ & $11.18 \pm 0.42^{\mathrm{a}, \mathrm{b}, \mathrm{c}}$ & $24.48 \pm 0.59^{\mathrm{a}, \mathrm{b}, \mathrm{c}}$ \\
\hline 18 & $12.39 \pm 0.86^{\mathrm{a}}$ & $1.32 \pm 0.29^{\mathrm{a}}$ & $35.06 \pm 0.26^{\mathrm{i}}$ & $10.55 \pm 0.62^{\mathrm{a}, \mathrm{b}}$ & $22.04 \pm 0.02^{\mathrm{a}}$ \\
\hline 19 & $7.72 \pm 1.07^{\mathrm{a}}$ & $0.57 \pm 0.01^{\mathrm{a}}$ & $23.98 \pm 1.75^{\mathrm{a}}$ & $17.16 \pm 0.93^{\mathrm{f}}$ & $30.65 \pm 0.74^{\mathrm{e}, \mathrm{f}}$ \\
\hline 20 & $9.84 \pm 0.83^{\mathrm{a}}$ & $1.01 \pm 0.02^{\mathrm{a}}$ & $29.92 \pm 1.45^{\mathrm{a}, \mathrm{b}, \mathrm{c}}$ & $12.37 \pm 0.52^{\mathrm{a}, \mathrm{b}, \mathrm{c}}$ & $22.36 \pm 1.56^{\mathrm{a}, \mathrm{b}}$ \\
\hline 21 & $9.65 \pm 0.62^{\mathrm{a}}$ & $1.06 \pm 0.02^{\mathrm{a}}$ & $29.02 \pm 0.33^{\mathrm{a}, \mathrm{b}, \mathrm{c}}$ & $13.05 \pm 0.56^{\mathrm{g}}$ & $22.86 \pm 1.07^{\mathrm{a}, \mathrm{b}}$ \\
\hline 22 & $10.53 \pm 0.83^{\mathrm{a}}$ & $1.10 \pm 0.01^{\mathrm{a}}$ & $28.94 \pm 1.48^{\mathrm{a}, \mathrm{b}, \mathrm{c}}$ & $14.44 \pm 0.67^{\mathrm{h}}$ & $28.33 \pm 0.79^{\mathrm{g}, \mathrm{c}, \mathrm{d}, \mathrm{e}, \mathrm{f}}$ \\
\hline 23 & $10.81 \pm 0.84^{\mathrm{a}}$ & $1.20 \pm 0.14^{\mathrm{a}}$ & $29.80 \pm 0.04^{\mathrm{a}, \mathrm{b}, \mathrm{c}}$ & $12.01 \pm 0.50^{\mathrm{a}, \mathrm{b}, \mathrm{c}}$ & $28.75 \pm 1.42^{\mathrm{c}, \mathrm{d}, \mathrm{e}, \mathrm{f}}$ \\
\hline 24 & $9.41 \pm 0.57^{\mathrm{a}}$ & $1.13 \pm 0.15^{\mathrm{a}}$ & $27.97 \pm 1.20^{\mathrm{a}, \mathrm{b}}$ & $13.33 \pm 0.52^{\mathrm{i}}$ & $32.27 \pm 0.73^{\mathrm{f}}$ \\
\hline 25 & $13.56 \pm 0.97^{\mathrm{a}}$ & $1.14 \pm 0.09^{\mathrm{a}}$ & $33.00 \pm 0.62^{j}$ & $12.20 \pm 0.33^{\mathrm{a}, \mathrm{b}, \mathrm{c}}$ & $21.92 \pm 1.15^{\mathrm{a}}$ \\
\hline 26 & $10.70 \pm 0.36^{\mathrm{a}}$ & $1.04 \pm 0.05^{\mathrm{a}}$ & $31.68 \pm 1.14^{\mathrm{b}, \mathrm{c}}$ & $12.05 \pm 0.56^{\mathrm{a}, \mathrm{b}, \mathrm{c}}$ & $26.95 \pm 1.20^{\mathrm{b}, \mathrm{c}, \mathrm{d}, \mathrm{e}}$ \\
\hline 27 & $11.39 \pm 0.91^{\mathrm{a}}$ & $1.35 \pm 0.31^{\mathrm{a}}$ & $32.73 \pm 1.40^{\mathrm{k}}$ & $10.82 \pm 1.15^{\mathrm{a}, \mathrm{b}, \mathrm{c}}$ & $25.46 \pm 1^{\mathrm{a}, \mathrm{b}, \mathrm{c}, \mathrm{d}}$ \\
\hline 28 & $7.94 \pm 0.42^{\mathrm{a}}$ & $0.94 \pm 0.01^{\mathrm{a}}$ & $29.00 \pm 0.36^{\mathrm{a}, \mathrm{b}, \mathrm{c}}$ & $18.20 \pm 0.90^{\mathrm{j}}$ & $28.80 \pm 0.98^{\mathrm{c}, \mathrm{d}, \mathrm{e}, \mathrm{f}}$ \\
\hline Mean & 10.98 & 1.17 & 31.10 & 12.64 & 26.07 \\
\hline
\end{tabular}


Table 2. (Continued) Fatty acid composition of butters ( $\%)$

\begin{tabular}{|c|c|c|c|c|c|}
\hline Butters & C18:2 & Others & SFA & MUFA & PUFA \\
\hline 1 & $2.97 \pm 0.05^{\mathrm{a}, \mathrm{b}, \mathrm{c}}$ & $3.08 \pm 0.50^{\mathrm{a}}$ & $69.52 \pm 0.59^{\mathrm{b}, \mathrm{c}, \mathrm{d}, \mathrm{e}}$ & $26.49 \pm 0.74^{\mathrm{b}, \mathrm{c}, \mathrm{d}}$ & $3.32 \pm 0.02^{\mathrm{a}}$ \\
\hline 2 & $3.08 \pm 0.11^{\mathrm{a}}$ & $3.11 \pm 3.42^{\mathrm{a}}$ & $67.81 \pm 2.71^{\mathrm{b}, \mathrm{c}, \mathrm{d}, \mathrm{e}}$ & $28.08 \pm 0.82^{\mathrm{d}, \mathrm{e}, \mathrm{f}, \mathrm{g}}$ & $3.42 \pm 0.29^{\mathrm{a}}$ \\
\hline 3 & $2.95 \pm 0.11^{\mathrm{a}}$ & $3.59 \pm 4.25^{\mathrm{a}}$ & $68.03 \pm 1.54^{\mathrm{b}, \mathrm{c}, \mathrm{d}, \mathrm{e}}$ & $28.15 \pm 0.70^{\mathrm{d}, \mathrm{e}, \mathrm{f}, \mathrm{g}}$ & $3.30 \pm 0.12^{\mathrm{a}}$ \\
\hline 4 & $2.84 \pm 0.48^{\mathrm{a}}$ & $3.09 \pm 0.06^{\mathrm{a}}$ & $68.00 \pm 2.00^{\mathrm{b}, \mathrm{c}, \mathrm{d}, \mathrm{e}}$ & $28.05 \pm 0.28^{\mathrm{d}, \mathrm{e}, \mathrm{f}, \mathrm{g}}$ & $3.21 \pm 0.28^{\mathrm{a}}$ \\
\hline 5 & $2.85 \pm 0.26^{\mathrm{a}}$ & $3.07 \pm 4.11^{\mathrm{a}}$ & $68.60 \pm 0.86^{\mathrm{b}, \mathrm{c}, \mathrm{d}, \mathrm{e}}$ & $27.51 \pm 0.73^{\mathrm{d}, \mathrm{e}, \mathrm{f}, \mathrm{g}}$ & $3.21 \pm 0.22^{\mathrm{a}}$ \\
\hline 6 & $2.63 \pm 0.33^{\mathrm{a}}$ & $3.26 \pm 4.61^{\mathrm{a}}$ & $66.44 \pm 0.93^{\text {a.b.c }}$ & $29.85 \pm 1.2^{\mathrm{e}, \mathrm{f}, \mathrm{g}, \mathrm{h}}$ & $3.03 \pm 0.22^{\mathrm{a}}$ \\
\hline 7 & $2.84 \pm 0.39^{\mathrm{a}}$ & $3.12 \pm 2.24^{\mathrm{a}}$ & $68.46 \pm 1.27^{\mathrm{b}, \mathrm{c}, \mathrm{d}, \mathrm{e}}$ & $27.61 \pm 0.87^{\text {d,e,f,g }}$ & $3.21 \pm 0.60^{\mathrm{a}}$ \\
\hline 8 & $2.80 \pm 0.49^{\mathrm{a}}$ & $3.00 \pm 1.25^{\mathrm{a}}$ & $64.56 \pm 0.81^{\mathrm{a} . \mathrm{b}}$ & $31.53 \pm 0.83^{\mathrm{h}, \mathrm{I}}$ & $3.22 \pm 0.29^{\mathrm{a}}$ \\
\hline 9 & $3.07 \pm 0.25^{\mathrm{a}}$ & $3.64 \pm 2.48^{\mathrm{a}}$ & $68.03 \pm 1.2^{\mathrm{b}, \mathrm{c}, \mathrm{d}, \mathrm{e}}$ & $27.92 \pm 1.37^{\mathrm{e}, \mathrm{fg}, \mathrm{h}}$ & $3.41 \pm 0.57^{\mathrm{a}}$ \\
\hline 10 & $1.24 \pm 0.02^{\mathrm{a}}$ & $4.89 \pm 2.75^{\mathrm{a}}$ & $72.72 \pm 2.1^{\mathrm{d}, \mathrm{e}}$ & $25.10 \pm 1.12^{\mathrm{b}, \mathrm{c}, \mathrm{d}}$ & $1.60 \pm 0.16^{\mathrm{a}}$ \\
\hline 11 & $2.55 \pm 0.33^{\mathrm{a}}$ & $2.98 \pm 0.47^{\mathrm{a}}$ & $69.87 \pm 1.25^{\mathrm{b}, \mathrm{c}, \mathrm{d}, \mathrm{e}}$ & $26.49 \pm 1.01^{\mathrm{b}, \mathrm{c}, \mathrm{d}}$ & $2.74 \pm 0.25^{\mathrm{a}}$ \\
\hline 12 & $2.72 \pm 0.21^{\mathrm{a}}$ & $3.64 \pm 1.06^{\mathrm{a}}$ & $69.54 \pm 0.77^{\mathrm{b}, \mathrm{c}, \mathrm{d}, \mathrm{e}}$ & $26.72 \pm 1.18^{\mathrm{b}, \mathrm{c}, \mathrm{d}, \mathrm{e}}$ & $3.06 \pm 0.41^{\mathrm{a}}$ \\
\hline 13 & $2.80 \pm 0.21^{\mathrm{a}}$ & $3.04 \pm 2.22^{\mathrm{a}}$ & $68.89 \pm 1.88^{\mathrm{b}, \mathrm{c}, \mathrm{d}, \mathrm{e}}$ & $27.23 \pm 0.33^{\mathrm{d}, \mathrm{e}, \mathrm{f}}$ & $2.99 \pm 0.15^{\mathrm{a}}$ \\
\hline 14 & $2.57 \pm 0.35^{\mathrm{a}}$ & $3.21 \pm 4.53^{\mathrm{a}}$ & $65.90 \pm 1.59^{\text {a.b. }}$ & $30.49 \pm 1.01^{\mathrm{g}, \mathrm{h}}$ & $2.93 \pm 0.16^{\mathrm{a}}$ \\
\hline 15 & $2.68 \pm 0.35^{\mathrm{a}}$ & $2.93 \pm 1.87^{\mathrm{a}}$ & $68.40 \pm 1.47^{\mathrm{b}, \mathrm{c}, \mathrm{d}, \mathrm{e}}$ & $27.89 \pm 0.96^{\mathrm{d}, \mathrm{e}, \mathrm{f}, \mathrm{g}}$ & $2.87 \pm 0.50^{\mathrm{a}}$ \\
\hline 16 & $2.81 \pm 0.35^{\mathrm{a}}$ & $3.01 \pm 0.15^{\mathrm{a}}$ & $69.13 \pm 0.79^{\mathrm{b}, \mathrm{c}, \mathrm{d}, \mathrm{e}}$ & $27.04 \pm 0.22^{\mathrm{c}, \mathrm{d}, \mathrm{e}, \mathrm{f}}$ & $3.18 \pm 0.25^{\mathrm{a}}$ \\
\hline 17 & $2.66 \pm 0.13^{\mathrm{a}}$ & $2.98 \pm 1.93^{\mathrm{a}}$ & $69.76 \pm 1.23^{\mathrm{b}, \mathrm{c}, \mathrm{d}, \mathrm{e}}$ & $26.55 \pm 0.96^{\mathrm{b}, \mathrm{c}, \mathrm{d}}$ & $2.85 \pm 0.41^{\mathrm{a}}$ \\
\hline 18 & $2.36 \pm 0.33^{\mathrm{a}}$ & $3.10 \pm 2.23^{\mathrm{a}}$ & $72.57 \pm 1.86^{\mathrm{d}, \mathrm{e}}$ & $24.05 \pm 0.24^{\mathrm{a}, \mathrm{b}, \mathrm{c}}$ & $2.54 \pm 0.26^{\mathrm{a}}$ \\
\hline 19 & $2.61 \pm 0.09^{\mathrm{a}}$ & $2.08 \pm 1.74^{\mathrm{a}}$ & $65.02 \pm 1.11^{\mathrm{a} . \mathrm{b}}$ & $31.56 \pm 0.97^{\mathrm{h}, \mathrm{I}}$ & $2.89 \pm 0.39^{\mathrm{a}}$ \\
\hline 20 & $2.31 \pm 0.29^{\mathrm{a}}$ & $2.27 \pm 0.39^{\mathrm{a}}$ & $72.94 \pm 1.51^{\mathrm{e}}$ & $23.14 \pm 0.5^{\mathrm{a}}$ & $2.69 \pm 0.33^{\mathrm{a}}$ \\
\hline 21 & $2.53 \pm 0.04^{\mathrm{a}}$ & $2.07 \pm 1.73^{\mathrm{a}}$ & $71.97 \pm 1.69^{\mathrm{c}, \mathrm{d}, \mathrm{e}}$ & $23.76 \pm 1.07^{\mathrm{a} b \mathrm{~b}}$ & $2.96 \pm 0.11^{\mathrm{a}}$ \\
\hline 22 & $2.89 \pm 0.62^{\mathrm{a}}$ & $3.03 \pm 2.74^{\mathrm{a}}$ & $65.89 \pm 2.98^{a . b}$ & $30.08 \pm 0.28^{\mathrm{e}, \mathrm{f}, \mathrm{g}, \mathrm{h}}$ & $3.32 \pm 0.07^{\mathrm{a}}$ \\
\hline 23 & $2.69 \pm 0.67^{\mathrm{a}}$ & $3.28 \pm 1.16^{\mathrm{a}}$ & $65.42 \pm 0.91^{\mathrm{a} . \mathrm{b}}$ & $30.62 \pm 0.87^{\mathrm{g}, \mathrm{h}}$ & $3.27 \pm 0.38^{\mathrm{a}}$ \\
\hline 24 & $2.88 \pm 0.45^{\mathrm{a}}$ & $3.03 \pm 1.64^{\mathrm{a}}$ & $61.87 \pm 1.55^{\mathrm{a}}$ & $34.08 \pm 0.28^{\mathrm{i}}$ & $3.30 \pm 0.04^{\mathrm{a}}$ \\
\hline 25 & $1.84 \pm 0.39^{\mathrm{a}}$ & $3.27 \pm 0.21^{\mathrm{a}}$ & $73.19 \pm 0.43^{\mathrm{e}}$ & $23.94 \pm 0.53^{a . b}$ & $2.22 \pm 0.02^{\mathrm{a}}$ \\
\hline 26 & $2.42 \pm 0.21^{\mathrm{a}}$ & $2.71 \pm 0.55^{\mathrm{a}}$ & $66.84 \pm 1.35^{\text {a.b.c.d }}$ & $28.76 \pm 1.07^{\mathrm{d}, \mathrm{e}, \mathrm{f}, \mathrm{g}, \mathrm{h}}$ & $2.82 \pm 0.07^{\mathrm{a}}$ \\
\hline 27 & $2.00 \pm 0.08^{\mathrm{a}}$ & $4.24 \pm 1.70^{\mathrm{a}}$ & $67.69 \pm 1.30^{\text {b.c.d.e }}$ & $28.49 \pm 0.69^{\mathrm{d}, \mathrm{e}, \mathrm{f}, \mathrm{g}, \mathrm{h}}$ & $2.43 \pm 0.45^{\mathrm{a}}$ \\
\hline 28 & $3.44 \pm 0.48^{\mathrm{a}}$ & $2.39 \pm 0.46^{\mathrm{a}}$ & $65.34 \pm 1.10^{\mathrm{a} . \mathrm{b}}$ & $30.13 \pm 0.35^{\mathrm{f}, \mathrm{g}, \mathrm{h}}$ & $3.59 \pm 0.19^{\mathrm{a}}$ \\
\hline Mean & 2.64 & 2.65 & 68.30 & 27.90 & 2.99 \\
\hline
\end{tabular}

\section{Thermal properties}

Melting point and solid fat content are among the most critical predictors of milk fat functionality. (Ortiz-Gonzalez et al., 2007). Although the entire DSC thermogram is the best expression of the melting properties, melting points calculated from DSC data are valuable information as melting points depend on triacylglycerol and fatty acid composition (Larsen et al., 2014). The melting point of milk fat is defined as the temperature at which milk fat becomes visually clear and free of crystals, and is approximately 32 to $36^{\circ} \mathrm{C}$ for "normal" milk fat (Ortiz-Gonzalez et al., 2007). 25 of 28 butters presented a typical DSC melting curve and had three endothermic peaks $\left(\mathrm{T}_{\mathrm{m} 1}, \mathrm{~T}_{\mathrm{m} 2}\right.$ and $\mathrm{T}_{\mathrm{m} 3}$ ) in accordance with literature data (Kashaninejad et al., 2017; Tomaszewska-Gras, 2013). All those 26 butter completely melted at this given range. However, B20 and 21 which were supplied from local markets were out of the mentioned range (Table 3). The endothermic region become narrower in the thermogram of those samples and melting peak shifted to lower temperatures. This may result from the dilution effect of some vegetable oils such as palm kernel oil. This result confirms the findings obtained from fatty acid composition analysis. Similar to our results, Ortiz-Gonzalez et al. (2007) reported a negative correlation between the content of C4$\mathrm{C} 10$ and the melting temperature of the butters.

The SFC of the butters was also investigated and the melting temperatures of the butters were aligned with SFC values. B20, B21, and B24 presented lower SFC values than those of the other butters at all temperatures (Table 3). At $20^{\circ} \mathrm{C}, \mathrm{B} 20$ and $\mathrm{B} 21$ had SFC values of 15.82 and 16.21 while the average value was 24.54. The reason for the sharp differences in SFC for the mentioned two butters may result from the infusion of various oils into butters. Chouinard et al. (1998) reported that supplemental calcium salts of vegetable oils decrease solid fat proportion at $5^{\circ} \mathrm{C}$. The solid fat content of the other butters was 
in parallel to the results recorded for butters in literature (Chouinard et al., 1998; Ortiz-Gonzalez et al., 2007; Queirós et al., 2016).

Table 3. (Continued) Melting profile and solid fat content (\%) of butters

\begin{tabular}{|c|c|c|c|c|c|}
\hline Butter & & $\begin{array}{l}\mathrm{T}_{\mathrm{m} 1} * \\
\left({ }^{\circ} \mathrm{C}\right)\end{array}$ & $\begin{array}{l}\mathrm{T}_{\mathrm{m} 2} \\
\left({ }^{\circ} \mathrm{C}\right)\end{array}$ & $\begin{array}{l}\mathrm{T}_{\mathrm{m} 3} \\
\left({ }^{\circ} \mathrm{C}\right)\end{array}$ & $\begin{array}{l}\mathrm{T}_{\mathrm{m}, \mathrm{end}} \\
\left({ }^{\circ} \mathrm{C}\right)\end{array}$ \\
\hline 1 & & $5.24 \pm 0.00^{\mathrm{a}, \mathrm{b}}$ & $14.39 \pm 0.00^{\mathrm{a}}$ & $30.75 \pm 0.00^{\mathrm{a}, \mathrm{b}}$ & $34.03 \pm 0.81^{b, c}$ \\
\hline 2 & & $4.84 \pm 0.00^{\mathrm{a}, \mathrm{b}}$ & $13.74 \pm 0.00^{a}$ & $31.16 \pm 0.00^{\mathrm{a}, \mathrm{b}, \mathrm{c}}$ & $34.23 \pm 0.45^{\mathrm{b}, \mathrm{c}}$ \\
\hline 3 & & $4.83 \pm 0.00^{\mathrm{a}, \mathrm{b}}$ & $14.48 \pm 0.00^{a}$ & $31.13 \pm 0.45^{\mathrm{a}, \mathrm{b}, \mathrm{c}}$ & $34.25 \pm 0.60^{\mathrm{b}, \mathrm{c}}$ \\
\hline 4 & & $5.49 \pm 0.00^{\mathrm{a}, \mathrm{b}}$ & $14.23 \pm 0.00^{a}$ & $31.32 \pm 0.00^{\mathrm{b}, \mathrm{c}, \mathrm{d}, \mathrm{e}}$ & $34.56 \pm 0.699^{b, c}$ \\
\hline 5 & & $5.32 \pm 0.00^{\mathrm{a}, \mathrm{b}}$ & $14.38 \pm 0.00^{a}$ & $31.32 \pm 0.00^{\mathrm{b}, \mathrm{c}, \mathrm{d}, \mathrm{e}}$ & $34.30 \pm 0.86^{\mathrm{b}, \mathrm{c}}$ \\
\hline 6 & & $4.89 \pm 0.00^{\mathrm{a}, \mathrm{b}}$ & $14.29 \pm 0.00^{a}$ & $32.00 \pm 0.00 \mathrm{~d}, \mathrm{e}, \mathrm{f}, \mathrm{g}$ & $35.60 \pm 0.00^{c}$ \\
\hline 7 & & $4.82 \pm 0.00^{\mathrm{a}, \mathrm{b}}$ & $13.97 \pm 0.00^{a}$ & $31.24 \pm 0.00^{\mathrm{a}, \mathrm{b}, \mathrm{c}, \mathrm{d}}$ & $33.33 \pm 2.08^{b, c}$ \\
\hline 8 & & $5.53 \pm 0.00^{\mathrm{a}, \mathrm{b}}$ & $14.38 \pm 0.00^{a}$ & $32.62 \pm 0.00 \mathrm{~g}$ & $35.52 \pm 0.00^{c}$ \\
\hline 9 & & $5.32 \pm 0.00^{\mathrm{a}, \mathrm{b}}$ & $14.68 \pm 0.04^{\mathrm{a}}$ & $31.83 \pm 0.28 \mathrm{c}, \mathrm{d}, \mathrm{e}, \mathrm{f}$ & $35.06 \pm 0.95^{\mathrm{b}, \mathrm{c}}$ \\
\hline 10 & & $5.06 \pm 0.25^{\mathrm{a}, \mathrm{b}}$ & $14.78 \pm 0.00^{a}$ & $31.48 \pm 0.00^{\mathrm{b}, \mathrm{c}, \mathrm{d}, \mathrm{e}, \mathrm{f}}$ & $35.71 \pm 0.86^{c}$ \\
\hline 11 & National & $4.95 \pm 0.12^{\mathrm{a}, \mathrm{b}}$ & $14.38 \pm 0.00^{a}$ & $32.16 \pm 0.00^{f, g}$ & $34.87 \pm 0.00^{\mathrm{b}, \mathrm{c}}$ \\
\hline 12 & Markets & $4.73 \pm 0.00^{\mathrm{a}, \mathrm{b}}$ & $14.28 \pm 0.00^{a}$ & $31.72 \pm 0.00 \mathrm{c}, \mathrm{d}, \mathrm{e}, \mathrm{f}$ & $34.89 \pm 0.50^{\mathrm{b}, \mathrm{c}}$ \\
\hline 13 & & $5.00 \pm 0.00^{\mathrm{a}, \mathrm{b}}$ & $14.32 \pm 0.00^{a}$ & $32.00 \pm 0.00 \mathrm{~d}, \mathrm{e}, \mathrm{f}, \mathrm{g}$ & $35.58 \pm 1.08^{c}$ \\
\hline 14 & & $4.70 \pm 0.03 \mathrm{a}, \mathrm{b}$ & $14.09 \pm 0.19^{a}$ & $31.07 \pm 0.00^{\mathrm{a}, \mathrm{b}, \mathrm{c}}$ & $33.93 \pm 0.74 \mathrm{~b}, \mathrm{c}$ \\
\hline 15 & & $3.93 \pm 0.00^{\mathrm{a}}$ & $14.17 \pm 0.00^{a}$ & $32.01 \pm 0.00^{\mathrm{d}, \mathrm{e}, \mathrm{f}, \mathrm{g}}$ & $33.89 \pm 0.70^{b, c}$ \\
\hline 16 & & $4.93 \pm 0.00^{\mathrm{a}, \mathrm{b}}$ & $14.42 \pm 0.00^{a}$ & $32.18 \pm 0.00^{f, g}$ & $34.83 \pm 0.43^{\mathrm{b}, \mathrm{c}}$ \\
\hline 17 & & $5.16 \pm 0.00^{\mathrm{a}, \mathrm{b}}$ & $14.32 \pm 0.08^{a}$ & $31.25 \pm 0.00^{\mathrm{a}, \mathrm{b}, \mathrm{c}, \mathrm{d}}$ & $34.17 \pm 0.59 b, c$ \\
\hline 18 & & $5.25 \pm 0.08 \mathrm{a}, \mathrm{b}$ & $14.55 \pm 0.00^{\mathrm{a}}$ & $31.45 \pm 0.00^{\mathrm{b}, \mathrm{c}, \mathrm{d}, \mathrm{e}, \mathrm{f}}$ & $34.66 \pm 1.26^{b, c}$ \\
\hline 19 & & $15.27 \pm 0.00^{\mathrm{e}}$ & $29.69 \pm 0.00^{a}$ & - & $34.18 \pm 0.00^{\mathrm{b}, \mathrm{c}}$ \\
\hline 20 & Local Markets & $9.74 \pm 1.24 \mathrm{~d}$ & $15.97 \pm 0.50^{a}$ & - & $28.58 \pm 1.02^{\mathrm{a}}$ \\
\hline 21 & & $10.98 \pm 0.00^{\mathrm{d}}$ & $16.47 \pm 0.00^{a}$ & - & $30.58 \pm 0.50^{\mathrm{a}, \mathrm{b}}$ \\
\hline 22 & & $5.75 \pm 0.75^{b, c}$ & $14.31 \pm 0.00^{a}$ & $32.16 \pm 0.00^{f, g}$ & $35.27 \pm 0.82$ \\
\hline 23 & & $4.59 \pm 0.33^{\mathrm{a}, \mathrm{b}}$ & $13.98 \pm 0.00^{\mathrm{a}}$ & $31.59 \pm 0.00 \mathrm{c}, \mathrm{d}, \mathrm{e}, \mathrm{f}$ & $34.28 \pm 0.55^{\mathrm{b}, \mathrm{c}}$ \\
\hline 24 & & $4.65 \pm 0.00^{\mathrm{a}, \mathrm{b}}$ & $13.64 \pm 0.17^{\mathrm{a}}$ & $32.07 \pm 0.01 \mathrm{e}, \mathrm{fg}$ & $35.82 \pm 1.18^{\mathrm{c}}$ \\
\hline 25 & Local & $5.84 \pm 0.00^{\mathrm{b}, \mathrm{c}}$ & $16.06 \pm 0.00^{a}$ & $32.67 \pm 0.00 \mathrm{~g}$ & $35.88 \pm 0.79 c$ \\
\hline 26 & Bazaars & $5.80 \pm 0.00^{b, c}$ & $14.95 \pm 0.00^{\mathrm{a}}$ & $30.50 \pm 0.00^{\mathrm{a}}$ & $36.80 \pm 0.00^{c}$ \\
\hline 27 & & $7.00 \pm 0.00^{c}$ & $15.15 \pm 0.00^{a}$ & $31.34 \pm 0.16^{\mathrm{b}, \mathrm{c}, \mathrm{d}, \mathrm{e}}$ & $34.13 \pm 0.72^{\mathrm{b}, \mathrm{c}}$ \\
\hline 28 & & $4.00 \pm 0.00^{\mathrm{a}}$ & $14.18 \pm 0.00^{\mathrm{a}}$ & $31.22 \pm 0.00^{\mathrm{a}, \mathrm{b}, \mathrm{c}, \mathrm{d}}$ & $36.00 \pm 0.25^{\mathrm{c}}$ \\
\hline Mean & & 5.84 & 15.08 & 31.61 & 34.27 \\
\hline
\end{tabular}

Table 3. (Continued) Melting profile and solid fat content (\%) of butters

\begin{tabular}{|c|c|c|c|c|c|}
\hline \multicolumn{2}{|c|}{ Butter } & $\begin{array}{l}\text { SFC } \\
5^{\circ} \mathrm{C}\end{array}$ & SFC & SFC & SFC \\
\hline 1 & & $74.55 \pm 0.75^{\mathrm{c}, \mathrm{d}, \mathrm{e}, \mathrm{f}, \mathrm{g}, \mathrm{h}, \mathrm{i}}$ & $63.79 \pm 0.69^{\mathrm{c}, \mathrm{d}, \mathrm{e}, \mathrm{f}, \mathrm{g}, \mathrm{h}}$ & $4166 \pm 0.46^{\mathrm{a}, \mathrm{b}, \mathrm{c}}$ & $2603+030^{\mathrm{a}, \mathrm{b}, \mathrm{c}, \mathrm{d}}$ \\
\hline 2 & & $73.62 \pm 0.14^{\mathrm{c}, \mathrm{d}, \mathrm{e}, \mathrm{f}, \mathrm{g}, \mathrm{h}}$ & $63.74 \pm 0.68^{\mathrm{c}, \mathrm{d}, \mathrm{e}, \mathrm{f}, \mathrm{g}, \mathrm{h}}$ & $41.07 \pm 0.15^{\mathrm{b}, \mathrm{c}}$ & $26.03+0.50^{\mathrm{a}, \mathrm{b}, \mathrm{c}, \mathrm{d}}$ \\
\hline 3 & & $7249 \pm 0.42^{\mathrm{c}, \mathrm{d}, \mathrm{e}, \mathrm{f}}$ & $61.88 \pm 0.38^{\mathrm{b}, \mathrm{c}, \mathrm{d}, \mathrm{e}, \mathrm{f}}$ & $41.33 \pm 0.24^{a, b, c}$ & $20.18 \pm 0.40^{a, b, b a}$ \\
\hline 4 & & $72.94 \pm 0.29^{\mathrm{c}, \mathrm{d}, \mathrm{e}, \mathrm{f}, \mathrm{g}}$ & $6171 \pm 026^{\mathrm{b}, \mathrm{c}, \mathrm{d}, \mathrm{e}, \mathrm{f}}$ & $4155+016^{\mathrm{a}, \mathrm{b}, \mathrm{c}}$ & \\
\hline 5 & & $73.65 \pm 0.33^{\mathrm{c}, \mathrm{d}, \mathrm{e}, \mathrm{f}, \mathrm{g}, \mathrm{h}}$ & $6295+031^{\mathrm{b}, \mathrm{c}, \mathrm{d}, \mathrm{e}, \mathrm{f}, \mathrm{g}}$ & $4174+022 \mathrm{a}, \mathrm{b}, \mathrm{c}$ & \\
\hline 6 & & & & & $25.65 \pm 0.15^{\mathrm{a}, 0, \mathrm{c}, \mathrm{a}}$ \\
\hline & & & $59.89 \pm 0.1 / 0$ & $40 . / / \pm 0.03^{, 0,0}$ & $27.56 \pm 0.26^{\mathrm{a}, \mathrm{b}, \mathrm{c}, \mathrm{d}}$ \\
\hline 7 & & $73.72 \pm 1.17^{\mathrm{c}, \mathrm{d}, \mathrm{e}, \mathrm{f}, \mathrm{g}, \mathrm{h}}$ & $62.86 \pm 1.08^{\mathrm{b}, \mathrm{c}, \mathrm{d}, \mathrm{e}, \mathrm{e}, \mathrm{g}}$ & $41.43 \pm 0.69^{\mathrm{a}, \mathrm{b}, \mathrm{c}}$ & $26.72 \pm 0.45^{\mathrm{a}, \mathrm{b}, \mathrm{c}, \mathrm{d}}$ \\
\hline 8 & & $75.36 \pm 0.00^{\mathrm{d}, \mathrm{e}, \mathrm{f}, \mathrm{g}, \mathrm{h}, \mathrm{i}}$ & $64.56 \pm 0.00^{\mathrm{d}, \mathrm{e}, \mathrm{f}, \mathrm{g}, \mathrm{h}}$ & $42.52 \pm 0.00^{c}$ & $26.84 \pm 0.00^{\mathrm{a}, \mathrm{b}, \mathrm{c}, \mathrm{d}}$ \\
\hline 9 & & $75.40 \pm 0.36^{\mathrm{d}, \mathrm{e}, \mathrm{f}, \mathrm{g}, \mathrm{h}, \mathrm{i}}$ & $64.98 \pm 0.34^{\mathrm{e}, \mathrm{f}, \mathrm{g}, \mathrm{h}}$ & $44.78 \pm 0.25^{\mathrm{c}}$ & $28.06 \pm 0.16^{\mathrm{c}, \mathrm{d}}$ \\
\hline 10 & & $78.82 \pm 0.60^{\mathrm{h}, \mathrm{i}}$ & $70.37 \pm 0.58^{j}$ & $51.38 \pm 0.44^{\mathrm{d}}$ & $33.35 \pm 0.29^{e}$ \\
\hline 11 & National & $77.33 \pm 0.06^{\mathrm{f}, \mathrm{g}, \mathrm{h}, \mathrm{i}}$ & $67.79 \pm 0.06^{\mathrm{h}, \mathrm{i}, \mathrm{j}}$ & $47.40 \pm 0.03^{\mathrm{e}}$ & $30.39 \pm 0.28^{\mathrm{f}}$ \\
\hline 12 & Markets & $76.05 \pm 0.58^{\mathrm{f}, \mathrm{g}, \mathrm{h}, \mathrm{i}}$ & $66.02 \pm 0.54^{\mathrm{f}, \mathrm{g}, \mathrm{h}, \mathrm{i}}$ & $45.71 \pm 0.69^{c}$ & $30.03 \pm 0.25^{\mathrm{g}}$ \\
\hline 13 & & $75.69 \pm 1.26^{\mathrm{e}, \mathrm{fg}, \mathrm{h}, \mathrm{i}}$ & $65.57 \pm 1.19^{\mathrm{e}, \mathrm{f}, \mathrm{g}, \mathrm{h}, \mathrm{i}}$ & $45.42 \pm 0.00^{\mathrm{f}}$ & $30.28 \pm 0.58^{\mathrm{h}}$ \\
\hline 14 & & $73.99 \pm 0.63^{\mathrm{c}, \mathrm{d}, \mathrm{e}, \mathrm{f}, \mathrm{g}, \mathrm{h}}$ & $62.97 \pm 0.58^{\mathrm{b}, \mathrm{c}, \mathrm{d}, \mathrm{e}, \mathrm{f}, \mathrm{g}}$ & $40.65 \pm 0.36^{\mathrm{a}, \mathrm{b}, \mathrm{c}}$ & $26.07 \pm 0.22^{\mathrm{a}, \mathrm{b}, \mathrm{c}, \mathrm{d}}$ \\
\hline 15 & & $79.97 \pm 0.05^{\mathrm{i}}$ & $69.70 \pm 0.05^{\mathrm{i}, \mathrm{j}}$ & $46.38 \pm 0.36^{\mathrm{g}}$ & $29.60 \pm 0.05^{\mathrm{i}}$ \\
\hline 16 & & $78.15 \pm 2.15^{\mathrm{g}, \mathrm{h}, \mathrm{i}}$ & $67.87 \pm 2.06^{\mathrm{h}, \mathrm{i}, \mathrm{j}}$ & $46.85 \pm 1.48^{c}$ & $30.43 \pm 1.01^{\mathrm{d}}$ \\
\hline 17 & & $75.02 \pm 1.15^{\mathrm{d}, \mathrm{e}, \mathrm{f}, \mathrm{g}, \mathrm{h}, \mathrm{i}}$ & $64.42 \pm 1.06^{\mathrm{c}, \mathrm{d}, \mathrm{e}, \mathrm{f}, \mathrm{g}, \mathrm{h}}$ & $42.45 \pm 0.67^{\mathrm{a}, \mathrm{b}, \mathrm{c}}$ & $26.67 \pm 0.41^{\mathrm{a}, \mathrm{b}, \mathrm{c}, \mathrm{d}}$ \\
\hline 18 & & $74.74 \pm 1.53^{\mathrm{c}, \mathrm{d}, \mathrm{e}, \mathrm{f}, \mathrm{g}, \mathrm{h}, \mathrm{i}}$ & $67.38 \pm 0.10^{\mathrm{g}, \mathrm{h}, \mathrm{i}, \mathrm{j}}$ & $45.85 \pm 1.21^{\mathrm{h}}$ & $28.91 \pm 1.52^{\mathrm{j}}$ \\
\hline
\end{tabular}


Table 3. (Continued) Melting profile and solid fat content (\%) of butters

\begin{tabular}{|c|c|c|c|c|c|}
\hline Butter & & $\begin{array}{l}\text { SFC } \\
5^{\circ} \mathrm{C} \\
\end{array}$ & $\begin{array}{l}\text { SFC } \\
10^{\circ} \mathrm{C}\end{array}$ & $\begin{array}{l}\mathrm{SFC} \\
15^{\circ} \mathrm{C} \\
\end{array}$ & $\begin{array}{l}\text { SFC } \\
20^{\circ} \mathrm{C}\end{array}$ \\
\hline 19 & & $76.83 \pm 0.75^{\mathrm{f}, \mathrm{g}, \mathrm{h}, \mathrm{i}}$ & $63.25 \pm 0.69^{\mathrm{b}, \mathrm{c}, \mathrm{d}, \mathrm{e}, \mathrm{f}, \mathrm{g}}$ & $40.70 \pm 0.44^{\mathrm{a}, \mathrm{b}, \mathrm{c}}$ & $19.15 \pm 0.17^{\mathrm{a}, \mathrm{b}, \mathrm{c}, \mathrm{d}}$ \\
\hline 20 & Local Markets & $67.52 \pm 0.10^{\mathrm{a}, \mathrm{b}}$ & $54.86 \pm 0.09^{\mathrm{a}}$ & $36.79 \pm 0.07^{\mathrm{a}}$ & $15.82 \pm 0.05^{\mathrm{a}}$ \\
\hline 21 & & $65.56 \pm 0.17^{\mathrm{a}}$ & $53.74 \pm 0.14^{\mathrm{a}}$ & $38.48 \pm 0.11^{\mathrm{a}}$ & $16.21 \pm 0.05^{\mathrm{a}, \mathrm{b}}$ \\
\hline 22 & & $71.26 \pm 0.96^{\mathrm{c}, \mathrm{d}}$ & $58.98 \pm 0.84^{\mathrm{b}}$ & $40.25 \pm 0.59^{\mathrm{a}, \mathrm{b}, \mathrm{c}}$ & $27.11 \pm 0.41^{\mathrm{a}, \mathrm{b}, \mathrm{c}, \mathrm{d}}$ \\
\hline 23 & & $71.76 \pm 0.19^{\mathrm{c}, \mathrm{d}, \mathrm{e}}$ & $61.12 \pm 0.18^{\mathrm{b}, \mathrm{c}, \mathrm{d}, \mathrm{e}}$ & $40.27 \pm 0.12^{\mathrm{a}, \mathrm{b}, \mathrm{c}}$ & $26.37 \pm 0.44^{\mathrm{a}, \mathrm{b}, \mathrm{c}, \mathrm{d}}$ \\
\hline 24 & & $65.93 \pm 0.88^{\mathrm{a}}$ & $54.45 \pm 0.77^{\mathrm{a}}$ & $38.87 \pm 0.58^{\mathrm{a}, \mathrm{b}}$ & $27.67 \pm 0.43^{\mathrm{a}, \mathrm{b}, \mathrm{c}, \mathrm{d}}$ \\
\hline 25 & Local & $85.35 \pm 0.68^{j}$ & $78.53 \pm 0.70^{\mathrm{k}}$ & $62.91 \pm 0.59^{\mathrm{i}}$ & $38.45 \pm 0.36^{\mathrm{k}}$ \\
\hline 26 & Bazaars & $70.75 \pm 0.10^{\mathrm{b}, \mathrm{c}}$ & $60.34 \pm 0.20^{\mathrm{b}, \mathrm{c}, \mathrm{d}}$ & $42.79 \pm 0.15^{\mathrm{a}, \mathrm{b}, \mathrm{c}}$ & $26.12 \pm 0.05^{\mathrm{a}, \mathrm{b}, \mathrm{c}, \mathrm{d}}$ \\
\hline 27 & & $73.08 \pm 0.13^{\mathrm{c}, \mathrm{d}, \mathrm{e}, \mathrm{f}, \mathrm{g}}$ & $64.86 \pm 2.62^{\mathrm{d}, \mathrm{e}, \mathrm{f}, \mathrm{g}, \mathrm{h}}$ & $44.55 \pm 0.09^{\mathrm{a}, \mathrm{b}, \mathrm{c}}$ & $27.34 \pm 0.05^{\mathrm{b}, \mathrm{c}, \mathrm{d}}$ \\
\hline 28 & & $71.21 \pm 0.15^{\mathrm{c}, \mathrm{d}}$ & $60.37 \pm 0.68^{\mathrm{b}, \mathrm{c}, \mathrm{d}}$ & $39.15 \pm 0.14^{\mathrm{a}, \mathrm{b}, \mathrm{c}}$ & $24.54 \pm 0.30^{\mathrm{a}, \mathrm{b}, \mathrm{c}}$ \\
\hline Mean & & 74.05 & 63.53 & 43.34 & 26.93 \\
\hline
\end{tabular}

\section{CONCLUSION}

Physicochemical properties, oxidation level, fatty acid compositions, and solid fat content of butters supplied from the Gaziantep market were investigated and compared with the Turkish national standard. The moisture content of 20 of $28(71.42 \%)$, the fat content of 21 of $28(75.0 \%)$, the non-solid fat content of 27 of $28(96.42 \%)$ butters, and acidity of all butters were outside the Turkish national standard. The salt contents of all of the butters complied with the standards. 27 of $28(96.42 \%)$ butters had peroxide values higher than the threshold value of peroxide value $(3.00$ $\mathrm{mEqO}_{2} / \mathrm{kg}$ fat). The TBA value of butters was lower than $0.3 \mathrm{mg} \mathrm{MA} / \mathrm{kg}$ fat. The thermal behavior and fatty acid results showed similarities and differences among the investigated butters. As a consequence, greater control during manufacturing, storage and transportation of butter is required to improve the quality and acceptability of butters and to decrease the product doubts of consumers by providing standard quality

\section{ACKNOWLEDGEMENTS}

Gaziantep University, Gaziantep, Turkey, supported the investigation. The author would like to thank M.Sc. students Melike Çeliktürk and Sultan Avşar for their technical assistance.

\section{CONFLICT OF INTEREST}

Author declares no conflict of interest.

\section{REFERENCES}

Akgül, H. İ., Şengül, M., Ürkek, B., Kotan, T. E. (2021). Determination of physicochemical and microbiological properties and fatty acid composition of butter produced in Trabzon, Turkey. Acta Scientiarum. Technology, 43: e48905e48905.

Altun, İ., Andic, S., Tunçtürk, Y., Cecen, A., Findik, O. (2011). Some chemical characteristics of butters obtained from Van market. Kafkas Universitesi Veteriner Fakïltesi Dergisi, 17(4): 645648.

Anonymous (1997) American Oil Chemists' Society (AOCS) Official and Recommended Practices of the AOCS. 7th Edition, AOCS Press Publication, Champaign, USA.

Anonymous (1998) Official Method of Analysis. AOAC International. 15 $5^{\text {th }}$ Edition, Washington DC, the USA.

Anonymous (2001). Butter-Determination of moisture, non-fat solids and fat contents - Part 2: Determination of non-fat solids content. The European Standard EN ISO 3727-2:2001.

Anonymous (2007). On emergency measures suspending the use of E 128 Red $2 \mathrm{G}$ as food colour. L 195/8 EN Official Journal of the European Union, Commission Regulation (EC) No 884/2007 of 26 July 2007.

Anonymous (2008). Laying down detailed rules for the application of Council Regulation (EC) No 1255/1999 as regards methods for the analysis and quality evaluation of milk and milk products. Commission Regulation (EC) No 273/2008 of 5 March 2008.

Anonymous (2021a). Durum Tahmin Raporu Süt Ve Süt Ürünleri. Hazırlayan: Dr. Zeliha Yasan Ataseven, Tarımsal Ekonomi ve Politika 
Geliştirme Enstitüsü Müdürlügüu, Çankaya, Ankara.

Anonymous (2021b). Tereyağ1 Standard1 (2. Bask1) (TS 1331). ICS 67.100.20; 67.200.10, Türk Standardlar1 Enstitüsü, Necatibey Caddesi No. 112, Bakanliklar, Ankara.

Asha, A., Manjunatha, M., Rekha, R., Surendranath, B., Heartwin, P., Rao, J., Magdaline, E., Sinha, C. (2015). Antioxidant activities of orange peel extract in ghee (butter oil) stored at different storage temperatures. Journal of Food Science and Technology, 52(12): 8220-8227.

Atamer, M., Kaptan, N. (1982). Ankara'da Tüketime Sunulan Kahvaltılık Tereyağların Nitelikleri Üzerinde Araştırmalar. Gıda, 7(4): 189: 198.

Atamer, M. (1993). Tereyağz Teknolojisi. Ankara Üniversitesi Yayınları. Ankara, Türkiye. Yayın No: 1313.

Atamer, M., Sezgin, E. (1984). Tereyağlarında Lipolitik ve oksidatif bozulmaların saptanmasinda yararlanilan asit ve peroksid değerleri ile aroma arasındaki ilişki. Gıda, 9(6): 329-334.

Celik, S., Bakirci, I. (2000). A study on the physical and chemical properties of cookery-type butter. Pakistan Journal of Biological Sciences, 3(4): 596-598.

Chouinard, P., Girard, V., Brisson, G. (1998). Fatty acid profile and physical properties of milk fat from cows fed calcium salts of fatty acids with varying unsaturation. Journal of Dairy Science, 81(2): 471-481.

Çakmakçı, S., Çelik, K., Ertem, H., Çalişkan, H. (2020). Tereyaği olarak satilan yağlarda bazi hileler ve kalite özelliklerinin tespiti: Erzurum örneği. Gida, 45(4): 800-813.

Çavdar, H. K., Yanık, D. K., Gök, U., Göğüş, F. (2017). Optimisation of microwave-assisted extraction of pomegranate (Punica granatum L.) seed oil and evaluation of its physicochemical and bioactive properties. Food Technology and Biotechnology, 55(1): 86.

da Silva, C. S., Almeida, L. S., Lima, C. M. G., Pagnossa, J. P., Neto, M. R. T., Brito, M. S., Barbosa, A. A., Santana, R. F. (2020).
Physicochemical quality of bottled butter. Brazilian Journal of Development, 6(2): 8994-9002.

Demirkaya, A. (2013). Tereyağında tiyobarbiturik asit (TBA) testi ile lipid oksidasyonunun değerlendirilmesi. Atatürk Universitesi Veteriner Bilimleri Dergisi, 8(3): 237-240.

Demirkol, A., Guneser, O., Karagul Yuceer, Y. (2016). Volatile compounds, chemical and sensory properties of butters sold in Çanakkale. Journal of Agricultural Sciences, 22(1): 99-108.

Dervisoglu, M., Gul, O., Guvenc, D., Yazici, F., Atmaca, E., Aksoy, A. (2013). Evaluation of chemical and microbiological characteristics and fatty acid profiles of butter samples collected from the black sea region of Turkey. Asian Journal of Chemistry, 25(18): 10185.

Ergin, G. (1976). Erzurum Ve Kars Yöresi Tereyağlarinin Gazkromatografik Analizi. Atatürk Universitesi Ziraat Fakültesi Dergisi, 7(4): 1-16.

Findık, O., Andiç, S. (2017). Some chemical and microbiological properties of the butter and the butter oil produced from the same raw material. LWT-Food Science and Technology, 86: 233-239.

Flakelar, C. L., Luckett, D. J., Howitt, J. A., Doran, G., Prenzler, P. D. (2015). Canola (Brassica napus) oil from Australian cultivars shows promising levels of tocopherols and carotenoids, along with good oxidative stability. Journal of Food Composition and Analysis, 42: 179186.

George, D., Mallery, M. (2010). SPSS for Windows Step by Step: A Simple Guide and Reference, 17.0 update (10a ed.). Pearson, Boston, USA.

Haron, H., Hiew, I., Shahar, S., Michael, V., Ambak, R. (2020). A survey on salt content labeling of the processed food available in Malaysia. International Journal of Environmental Research and Public Health, 17(7): 2469.

Idoui,T., Rechak, H. and Zabayou, N. (2013). Microbial quality, physicochemical characteristics and fatty acid composition of a traditional butter made from goat milk. Annals Food Science and Technology, 14(1): 108-114. 
Kashaninejad, M., Razavi, S., Mazaheri Tehrani, M., Kashaninejad, M. (2017). Fatty acid composition, rheological and thermal properties of butter from sheep's and omega-3 cow's milks. Iranian Journal of Science and Technology, 13(3): 6679.

Koca, N., Kocaoglu-Vurma, N., Harper, W., Rodriguez-Saona, L. (2010). Application of temperature-controlled attenuated total reflectance-mid-infrared (ATR-MIR) spectroscopy for rapid estimation of butter adulteration. Food Chemistry, 121(3): 778-782.

Konkol, K. L., Rasmussen, S. C. (2015). An ancient cleanser: soap production and use in antiquity, In: Chemical Technology in Antiquity, Rasmussen, S. C. (chief ed.), ACS Publications, USA, pp. 245-266.

Larsen, M., Andersen, K., Kaufmann, N., Wiking, L. (2014). Seasonal variation in the composition and melting behavior of milk fat. Joumal of Dairy Science, 97(8): 4703-4712.

Méndez-Cid, F. J., Centeno, J. A., Martínez, S., Carballo, J. (2017). Changes in the chemical and physical characteristics of cow's milk butter during storage: Effects of temperature and addition of salt. Journal of Food Composition and Analysis, 63: 121-132.

Ortiz-Gonzalez, G., Jimenez-Flores, R., Bremmer, D., Clark, J., DePeters, E., Schmidt, S., Drackley, J. (2007). Functional properties of butter oil made from bovine milk with experimentally altered fat composition. Journal of Dairy Science, 90(11): 5018-5031.

Queirós, M. S., Grimaldi, R., Gigante, M. L. (2016). Addition of olein from milk fat positively affects the firmness of butter. Food Research International, 84: 69-75.

Samet-Bali, O., Ayadi, M., Attia, H. (2009). Traditional Tunisian butter: Physicochemical and microbial characteristics and storage stability of the oil fraction. LWT-Food Science and Technology, 42(4): 899-905.

Sevmiş, E., Andiç, S., Şehriban, O. (2020) Comparison of the compositions of butter and butter oil produced in the province of Hakkari. Yüュüncü Yal Üniversitesi Tarm Bilimleri Dergisi, 30: 928-937.

Tomaszewska-Gras, J. (2013). Melting and crystallization DSC profiles of milk fat depending on selected factors. Journal of Thermal Analysis and Calorimetry, 113(1): 199-208.

Tomaszewska-Gras, J. (2016). DSC coupled with PCA as a tool for butter authenticity assessment. Journal of Thermal Analysis and Calorimetry, 126(1): 61-68.

Wilbey, R. A. (2009). Butter, In: Dairy fat and related products, Tamime, A. Y. (Chief ed.), WileyBlackwell Publishing, the UK, pp. 86-107. 